\title{
JOINT INVERSION OF COMPACT OPERATORS
}

\author{
by \\ James Ford
}

\author{
A thesis \\ submitted in partial fulfillment \\ of the requirements for the degree of \\ Master of Science in Mathematics \\ Boise State University
}

August 2017 
(C) 2017

James Ford

ALL RIGHTS RESERVED 
BOISE STATE UNIVERSITY GRADUATE COLLEGE

DEFENSE COMMITTEE AND FINAL READING APPROVALS

of the thesis submitted by

James Ford

Thesis Title: Joint Inversion of Compact Operators

Date of Final Oral Examination: 24 May 2017

The following individuals read and discussed the thesis submitted by student James Ford, and they evaluated his presentation and response to questions during the final oral examination. They found that the student passed the final oral examination.

Jodi Mead, Ph.D. C Chair, Supervisory Committee

John Bradford, Ph.D. Member, Supervisory Committee

Grady Wright, Ph.D. Member, Supervisory Committee

The final reading approval of the thesis was granted by Jodi Mead, Ph.D., Chair of the Supervisory Committee. The thesis was approved by the Graduate College. 
dedicated to Cassidy Carroll 


\section{ACKNOWLEDGMENTS}

First, I would like to thank my advisor Dr. Jodi Mead, who introduced me to inverse problems and academic research. Her patience and insights throughout my time at Boise State University have been invaluable. I would also like to extend my gratitude to the other members of my thesis committee, Dr. Grady Wright and Dr. John Bradford. All of them have been continually encouraging and are exemplary researchers whose work I try to model my own after.

My sincere thanks goes out to the Department of Mathematics whose financial support has allowed me to attend a number of impactful conferences and workshops during my time as a student. I also thank my fellow classmates in the mathematics

program. Enduring this experience together made it more productive and vastly more enjoyable.

I thank my family: My parents Ann and Chris, who fostered my intellectual curiosity from a young age and have always supported my decisions; and my brother Ben, whose relentless competition has continuously pushed me to become better.

Finally, I wish to thank Cass, whose impact on my life is immeasurable. Her unending support has made all of this possible. 


\begin{abstract}
The first mention of joint inversion came in [22], where the authors used the singular value decomposition to determine the degree of ill-conditioning in inverse problems. The authors demonstrated in several examples that combining two models in a joint inversion, and effectively stacking discrete linear models, improved the conditioning of the problem. This thesis extends the notion of using the singular value decomposition to determine the conditioning of discrete joint inversion to using the singular value expansion to determine the well-posedness of joint linear operators. We focus on compact linear operators related to geophysical, electromagnetic subsurface imaging.

The operators are based on combining Green's function solutions to differential equations representing different types of data. Joint operators are formed by extending the concept of stacking matrices to one of combining operators. We propose that the effectiveness of joint inversion can be evaluated by comparing the decay rate of the singular values of the joint operator to those from the individual operators. The joint singular values are approximated by an extension of the Galerkin method given in $[9,18]$. The approach is illustrated on a one-dimensional ordinary differential equation where slight improvement is observed when naively combining differential equations. Since this approach relies primarily on the differential equations representing data, it provides a mathematical framework for determining the effectiveness of joint inversion methods. It can be extended to more realistic differential equations in order to better inform the design of field experiments.
\end{abstract}




\section{TABLE OF CONTENTS}

ABSTRACT $\ldots \ldots \ldots \ldots \ldots \ldots \ldots \ldots \ldots \ldots \ldots \ldots \ldots \ldots \ldots \ldots \ldots$

LIST OF FIGURES $\ldots \ldots \ldots \ldots \ldots \ldots \ldots \ldots \ldots \ldots \ldots \ldots \ldots \ldots \ldots \ldots$

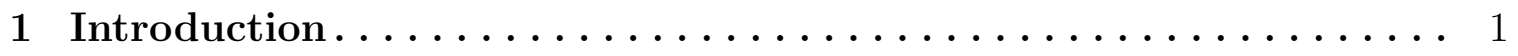

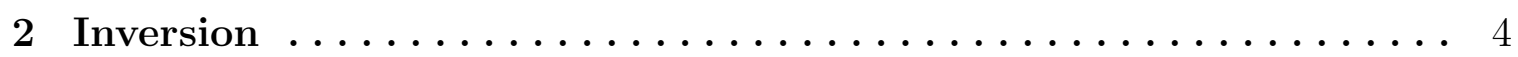

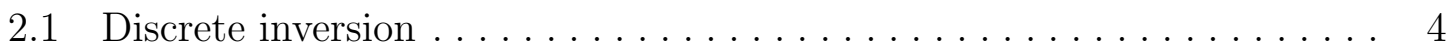

2.1.1 Linear problems $\ldots \ldots \ldots \ldots \ldots \ldots \ldots \ldots \ldots \ldots \ldots$

2.1.2 Singular value decomposition $\ldots \ldots \ldots \ldots \ldots \ldots \ldots \ldots$

2.1.3 Conditioning and Regularization ................ 8

2.2 Inversion with compact operators . . . . . . . . . . . . . . 10

2.2 .1 Green's functions $\ldots \ldots \ldots \ldots \ldots \ldots \ldots \ldots \ldots \ldots \ldots$

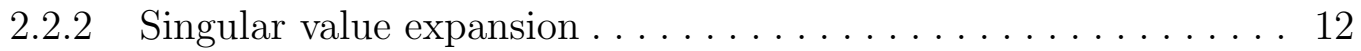

2.2 .3 Conditioning and ill-posedness $\ldots \ldots \ldots \ldots \ldots \ldots \ldots \ldots$

$2.2 .4 \quad$ Regularization ......................... 18

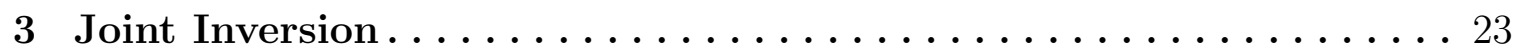

3.1 Discrete joint inversion . . . . . . . . . . . . . . . . . 23

3.1 .1 Linear problems $\ldots \ldots \ldots \ldots \ldots \ldots \ldots \ldots \ldots \ldots \ldots$

3.1 .2 Green's functions example.................... 24

3.2 Joint inversion with compact operators $\ldots \ldots \ldots \ldots \ldots \ldots \ldots \ldots$ 
3.2 .1 Joint operators $\ldots \ldots \ldots \ldots \ldots \ldots \ldots \ldots \ldots \ldots \ldots \ldots$

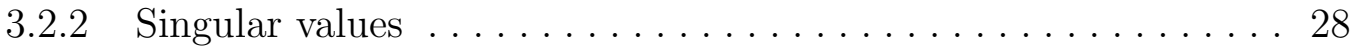

3.2.3 Approximation of SVE with SVD . . . . . . . . . . . . . 32

4 Application with compact operators $\ldots \ldots \ldots \ldots \ldots \ldots \ldots \ldots$

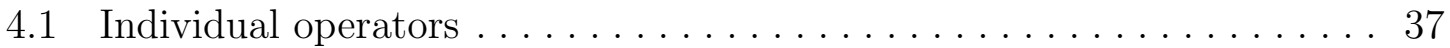

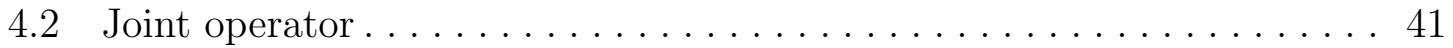

4.3 Joint singular values . . . . . . . . . . . . . . . . . 44

5 Conclusions and future work $\ldots \ldots \ldots \ldots \ldots \ldots$

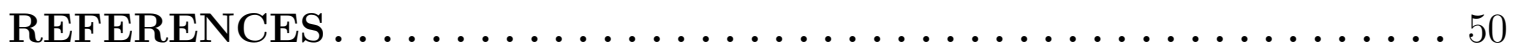




\section{LIST OF FIGURES}

1.1 Electrical resistivity setup [4] (left) and ground penetrating radar [5]

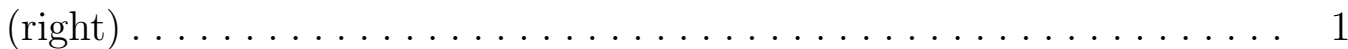

2.1 Singular values of A on a semi-log scale (a), and a log-log scale (b) . . . 18

3.1 Singular values of individual operators and joint operator . . . . . . 26

3.2 Parametric curve defined by $C(\cos k x)$ for $k \in[-5,5] \ldots \ldots \ldots$

4.1 Singular values of individual operators . . . . . . . . . . . . . . . 42

4.2 Analytical singular values and Galerkin method approximations using orthonormal box functions . . . . . . . . . . . . . . . . . 45

4.3 Approximate singular values of the joint operator $C$ on a semi-log scale (a), and a $\log -\log$ scale $(b) \ldots \ldots \ldots \ldots \ldots \ldots$

4.4 Approximate singular values of the individual operators $A$ and $B$, and the joint operator $C$ on a semi-log scale (a), and a log-log scale (b) . . 47 


\section{CHAPTER 1}

\section{INTRODUCTION}

This thesis is motivated by electromagnetic imaging of the Earth's subsurface using two data collection methods - ground penetrating radar (GPR) and electrical resistivity (ER), both depicted in Figure 1.1. The ER survey on the left uses electrodes to introduce and measure current within the Earth's subsurface, from which the electrical conductivity can be estimated within a localized region. The GPR survey on the right involves a transmitter emitting radar pulses into the subsurface. The energy reflected back by dielectric interfaces within the subsurface is recorded by the

receiver, and conductivity can also be inferred. Joint inversion of these data can give conductivity estimates on a vast range of frequency bands from $10^{0}$ to $10^{9} \mathrm{~Hz}$. A comprehensive introduction to geophysical electromagnetic imaging can be found in $[19,23]$.
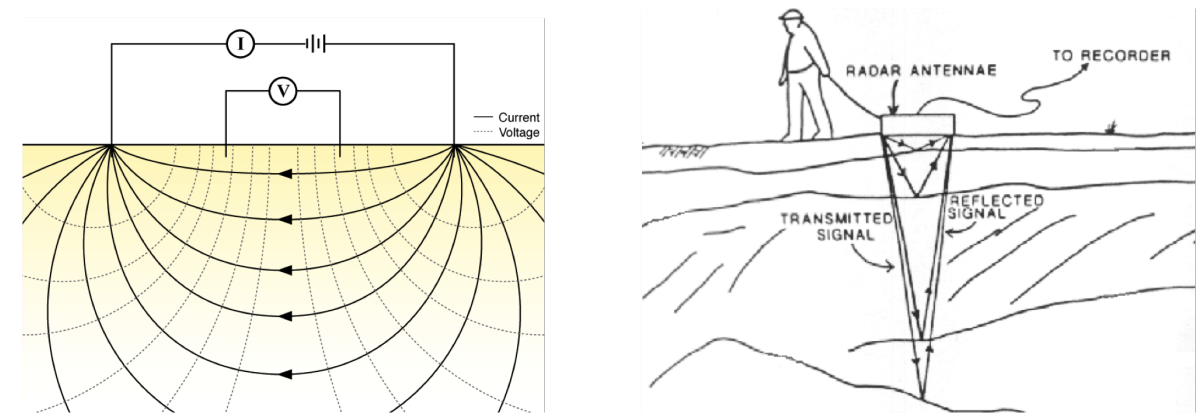

Figure 1.1: Electrical resistivity setup [4] (left) and ground penetrating radar [5] (right) 
Maxwell's equations govern the behavior of both ER and GPR, relating input sources to observable responses [14]. If we assume the domain we are interested in imaging is linear and isotropic, Maxwell's equations can be decoupled into the wave equation and the diffusion equation. Under these assumptions, the damped wave equation governs GPR:

$$
\varepsilon \frac{\partial^{2} E}{\partial t^{2}}+\sigma \frac{\partial E}{\partial t}=\frac{1}{\mu} \nabla^{2} E+f,
$$

where $E$ is the electric field component, $f$ is the source; while the parameters $\mu, \varepsilon$, and $\sigma$ are scalar functions of space representing magnetic permeability, dielectric permittivity and conductivity, respectively. The forward problem takes the parameters as input and produces the electric field as a function of space and time. The inverse problem takes discrete measurements of the electric field and produces parameter estimates that can be used to image the Earth's subsurface.

Alternatively, the diffusion equation governs ER and assumes no time dependence of the electric field:

$$
-\nabla \cdot \sigma \nabla \phi=\nabla \cdot J_{s}
$$

where $J_{s}$ is the source current density and $\phi$ is the electric field potential. Similar to GPR, given discrete measurements of the electric field potential, the conductivity can be estimated through an inversion.

In the early 1900s, French mathematician Jacques Hadamard introduced the concept of a well-posed problem of mathematical physics [21]. He proposed that mathematical models of physical phenomena should have the properties that for every set of inputs, a solution exists, the solution is unique, and the problem is stable. i.e., the solutions behavior changes continuously with the inputs. A problem that does not satisfy these conditions is said to be ill-posed. Problems failing to meet 
these conditions arise with great frequency in many areas of science and engineering, including electromagnetic subsurface imaging [11]. Since inverse problems are notoriously ill-posed, approaches to solving them will be the focus of this thesis.

The primary tools we will use for the analysis of inverse problems are the singular value decomposition (SVD) and its continuous analogue, the singular value expansion (SVE). We begin in Chapter 2 with a brief introduction to the solutions of discrete inverse problems using the SVD. Green's functions are then introduced since they continuously describe the solution of differential equations as a function of unknown parameters or sources. The corresponding operators are consequently decomposed using the SVE. A simple example is given with the focus on the behaviors of the corresponding singular values. Tikhonov regularization in the context of the SVE is also described.

In Chapter 3 we extend the concepts in Chapter 2 to joint inversion. Analysis of discrete joint inverse problems is somewhat straightforward because we simply view two models as one stacked matrix. However, in the continuous setting, the joint operator requires more careful consideration. Therefore we extend notions of Tikhonov regularization found in [6] to the more general direct sum of integral operators on Hilbert spaces, as in [8]. Approaches to calculating the continuous joint singular values are then given, and they include both analytical and numerical methods. Finally, in Chapter 4 we illustrate the approaches on a one-dimensional ODE. The singular values of the individual operators are found analytically, while those for the joint operator are found numerically. The singular values are compared and slight improvement with joint inversion is observed qualitatively through the decay rate of the singular values. 


\section{CHAPTER 2}

\section{INVERSION}

\subsection{Discrete inversion}

Data collection is typically represented as a finite number of measurements taken at discrete points in space and time. It is therefore natural to first consider a discrete inverse problem, with observations denoted $b=\left(b_{1}, b_{2}, \ldots b_{m}\right)$ and parameters we seek to recover denoted $x=\left(x_{1}, x_{2}, \ldots x_{n}\right)$. Then, with $g$ being the model relating $x$ and $b$, we attempt to solve the system $g(x)=b$, or

$$
\left[\begin{array}{c}
g_{1}\left(x_{1}, x_{2}, \ldots, x_{n}\right) \\
g_{2}\left(x_{1}, x_{2}, \ldots, x_{n}\right) \\
\vdots \\
g_{m}\left(x_{1}, x_{2}, \ldots, x_{n}\right)
\end{array}\right]=\left[\begin{array}{c}
b_{1} \\
b_{2} \\
\vdots \\
b_{m}
\end{array}\right]
$$

for the parameter vector $x$. As previously mentioned, such problems may be ill-posed. A careful analysis of the model $g$ can reveal if the problem has any solutions, a unique solution and if it is stable. Such insights can inform methods that allow us to proceed in any case.

Discrete inverse problems are well studied and general introductions to them can be found in $[1,10,11,12]$. This section gives a brief overview of discrete inverse 
problems as well as the methods used to analyze and solve them. This background will set the stage for the continuous analogues developed in this thesis.

\subsubsection{Linear problems}

The simplest case of discrete inverse problems occurs when the model and parameters are related linearly. That is,

$$
\left[\begin{array}{c}
g_{1}\left(x_{1}, x_{2}, \ldots, x_{n}\right) \\
g_{2}\left(x_{1}, x_{2}, \ldots, x_{n}\right) \\
\vdots \\
g_{m}\left(x_{1}, x_{2}, \ldots, x_{n}\right)
\end{array}\right]=\left[\begin{array}{c}
a_{11} x_{1}+a_{12} x_{2}+\cdots+a_{1 n} x_{n} \\
a_{21} x_{1}+a_{22} x_{2}+\cdots+a_{2 n} x_{n} \\
\vdots \\
a_{m 1} x_{1}+a_{m 2} x_{2}+\cdots+a_{m n} x_{n}
\end{array}\right]
$$

In this case, the model can be represented as matrix, and we solve $A x=b$ to recover the parameters. We will devote our attention to linear problems. The assumption of linearity is not so restrictive, since most non-linear methods involve iteratively solving a linear problem. Therefore this thesis is devoted to analyzing and solving linear problems.

\subsubsection{Singular value decomposition}

The primary tool used for the analysis of linear inverse problems is the singular value decomposition (SVD). This algebraic decomposition represents a special case of the singular value expansion (SVE), which will be discussed in 2.2.2.

Theorem 2.1.1 (Singular Value Decomposition). Let $A \in \mathbb{R}^{m \times n}$ be a matrix of rank $r$. Then, there exist orthogonal matrices $U \in \mathbb{R}^{m \times m}$ and $V \in \mathbb{R}^{n \times n}$ such that 


$$
A=U \Sigma V^{T}, \quad \Sigma=\left(\begin{array}{cc}
\Sigma_{r} & 0 \\
0 & 0
\end{array}\right)
$$

where $\Sigma \in \mathbb{R}^{m \times n}, \Sigma_{r}=\operatorname{diag}\left(\sigma_{1}, \sigma_{2}, \ldots \sigma_{r}\right)$ and

$$
\sigma_{1} \geq \sigma_{2} \geq \ldots \sigma_{r}>0
$$

Letting $v_{j}$ and $u_{j}$ be the jth columns of $V$ and $U$ respectively, we have that

$$
\begin{array}{cl}
A v_{j}=\sigma_{j} u_{j}, \quad A^{T} u_{j}=\sigma_{j} v_{j}, & \text { for } j=1, \ldots, n \\
A^{T} u_{j}=0, & \text { for } j=n+1, \ldots, m
\end{array}
$$

Proof. See [15].

This decomposition is enormously useful. For example, the norm of a vector is invariant under multiplication by an orthogonal matrix. So when considering $A x=$ $U \Sigma V^{T} x$, the scaling affect of the model can be isolated to $\Sigma$. This allows us to classify if solutions will exist and the conditioning of the model. Additionally, this can yield insights into the importance of each parameter, $x_{i}$. Parameters associated with large singular values will have a significantly greater influence on the outcome of the forward problem and can therefore be classified as more important [22].

In the case of a square matrix with full rank, $r=m=n$, we can very easily formulate the inverse of $A$ using the SVD, and therefore the solution to $A x=b$ :

$$
A^{-1}=V \Sigma^{-1} U^{T}, \quad x=V \Sigma^{-1} U^{T} b .
$$

However, most applications result in non-square and or non-full rank matrices and 
the inverse does not exist. In the case of full rank, overdetermined systems we can find the least squares solution to the problem; that is, the parameter vector that minimizes

$$
\|b-A x\|_{2}^{2}
$$

The solution estimate in this case is

$$
\hat{x}=\left(A^{T} A\right)^{-1} A^{T} b
$$

The generalized inverse allows us to define the solution estimate in terms of the SVD when A is not invertible. We construct the generalized inverse by first defining $\Sigma^{\dagger} \in \mathbb{R}^{n \times m}$ as

$$
\Sigma^{\dagger}=\left(\begin{array}{cc}
\Sigma_{r}^{-1} & 0 \\
0 & 0
\end{array}\right)
$$

Then we can define the generalized inverse $A^{\dagger}$ as

$$
A^{\dagger}=V \Sigma^{\dagger} U^{T}
$$

Further, we can define a generalized (or pseudo) inverse solution $x_{\dagger}$ by

$$
\begin{aligned}
x_{\dagger} & =A^{\dagger} b \\
& =V \Sigma^{\dagger} U^{T} b \\
& =\sum_{i=1}^{r} \frac{u_{i}^{T} b}{\sigma_{i}} v_{i} .
\end{aligned}
$$

It can be shown that $x_{\dagger}$ is the solution that minimizes $\|b-A x\|_{2}^{2},[1]$. 


\subsubsection{Conditioning and Regularization}

As stated previously, the third property that a well-posed problem should possess is stability, i.e., the solutions behavior changes continuously with the initial conditions. Typically, discrete problems are solved computationally. Therefore, the property we are interested in becomes numerical stability or conditioning. Given the SVD of a model, we are able to determine to what degree the inverse solution will be affected by perturbations in the inputs. The metric by which ill-conditioning is measured is called the condition number.

Definition 2.1.1. (Condition number) The condition number of $A$ is defined to be $\kappa(A)=\sigma_{1} / \sigma_{r}$, where $r=\operatorname{rank}(A)$.

As can be seen above, the generalized solution, $x_{\dagger}$, is dependent on the inverse

of the singular values of $A$. If $\sigma_{1}^{-1}<<\sigma_{p}^{-1}$, for some $p$ where $1<p \leq r$, then the portion of the summation

$$
\sum_{i=p}^{r} \frac{u_{i}^{T} b}{\sigma_{i}} v_{i}
$$

will dominate the solution. The ratio of the largest singular value to the smallest quantifies this effect. For $\kappa(A)$ large, the values in $b$ associated with $\sigma_{r}$ will be scaled by a much greater amount than the values associated with $\sigma_{1}$. Therefore, small perturbations in $b$ can result in large changes to the solution. There are a vast number of regularization methods for addressing this issue of ill-conditioning $[1,11$, 10]. We will focus on two in particular, the truncated singular value decomposition and Tikhonov regularization.

Ill-conditioning occurs when $\kappa(A)=\sigma_{1} / \sigma_{r}$ is large. This encourages us to truncate the smallest singular values of $A$ up to $\sigma_{k}$ for some $k<r$, so that $\sigma_{1} / \sigma_{k}<<\sigma_{1} / \sigma_{r}$. More precisely, let 


$$
A_{k}=U \Sigma V^{T}, \quad \Sigma=\left(\begin{array}{cc}
\Sigma_{k} & 0 \\
0 & 0
\end{array}\right),
$$

where $\Sigma_{k}=\operatorname{diag}\left(\sigma_{1}, \sigma_{2}, \ldots, \sigma_{k}\right)$. Then the solution using this truncated matrix is

$$
\begin{aligned}
x_{\dagger}^{k} & =A_{k}^{\dagger} b \\
& =\sum_{i=1}^{k} \frac{u_{i}^{T} b}{\sigma_{i}} v_{i} .
\end{aligned}
$$

The matrix $A_{k}$ is the best rank $k$ approximation to $A$ [10]. If we cut off a small number singular values, we will have a very good approximation of the original matrix, and our inverse solution will be less susceptible to perturbations in $b$. The choice of truncation value is challenging and not within the scope of this thesis. Though, in general, it is advantageous to keep as many singular values as possible, while maintaining stability.

Tikhonov regularization is another method of combating ill-conditioning. Here, we solve a damped least squares problem

$$
\min _{x}\|b-A x\|_{2}^{2}+\alpha^{2}\|x\|_{2}^{2}
$$

or equivalently

$$
\min _{x}\left\|\left[\begin{array}{l}
b \\
0
\end{array}\right]-\left[\begin{array}{l}
A \\
\alpha I
\end{array}\right] x\right\|_{2}^{2} .
$$

For large enough $\alpha$, this damped least squares problem always has a solution. The solution $x_{\dagger}^{\alpha}$, is dependent on $\alpha$ and can be expressed in terms of the SVD of $A$ [1]. Namely,

$$
x_{\dagger}^{\alpha}=\sum_{i=1}^{r} \frac{\sigma_{i}^{2}}{\sigma_{i}^{2}+\alpha^{2}} \frac{u_{i}^{T} b}{\sigma_{i}} v_{i} .
$$


By writing the solution in this form, we see that the contribution of singular values smaller than alpha are damped out. However, we still have the challenge of choosing an $\alpha$ that improves the conditioning, but does not significantly change the problem. Methods for choosing the regularization parameter include the discrepancy principle, L-curve and generalized cross validation (GCV) [1], but these fall outside the scope of this thesis.

\subsection{Inversion with compact operators}

In this section, we extend the notions in 2.1 to continuous functions and compact linear operators. Since we are interested in physical phenomena modeled by differential equations, we wish to consider operators with some physical significance. Green's functions solutions of inhomogeneous differential equations determine the response of the physical system for given a source. The integral operators corresponding to Green's functions are therefore the compact operators we will investigate. They provide a physically motivated, yet abstract paradigm in which we may consider inverse problems. A thorough discussion of Green's functions and their applications can be found in [3].

\subsubsection{Green's functions}

Let $\mathcal{L}_{A}=\mathcal{L}_{A}(t)$ be a linear differential operator. Then the corresponding Green's function, $K_{A}(t, s)$ satisfies

$$
\mathcal{L}_{A} K_{A}(t, s)=\delta(t-s)
$$

where $\delta$ denotes the delta function. We solve for the Green's function as a function of $s$ over the same domain $\Omega$ as $t$ to determine the response of the system to an impulse 
(delta function).

Given the Green's function, we can find the solution to the inhomogeneous equation $\mathcal{L}_{A} u(t)=f(t)$ for a given specific, $f$. This is accomplished by integrating both sides of equation (2.1) against $f(s)$ over $s$ in the domain $\Omega$,

$$
\int_{\Omega} \mathcal{L}_{A} K_{A}(t, s) f(s) d s=\int_{\Omega} \delta(t-s) f(s) d s .
$$

Since $\mathcal{L}_{A}$ is a an operator acting only on $t$, it can be moved outside of the integral. Therefore we have,

$$
\mathcal{L}_{A}\left(\int_{\Omega} K_{A}(t, s) f(s) d s\right)=f(t)
$$

Thus the solution to $\mathcal{L}_{A} u(t)=f(t)$ for a given source $f$ is

$$
u(t)=\int_{\Omega} K_{A}(t, s) f(s) d s
$$

In other words, integrating the Green's function against the source gives the response of the system to that source.

If we define the operator $A$ to be

$$
A h(t)=\int_{\Omega} K_{A}(t, s) h(s) d s
$$

then $A$ maps a source $h$ to the response of the system. In a physically motivated scenario, this equation describes how a model $A$ relates sources $h(t)$ to a response $u(t)$. The forward problem predicts the response for a given source, while the inverse problem recovers a source, given the response. To analyze Green's functions, we must 
first work through some general results concerning compact operators.

\subsubsection{Singular value expansion}

When considering discrete inverse problems, the singular value decomposition is the tool of choice for rigorous analysis of the problem and its least squares solution. The continuous extension of this tool is the singular value expansion (SVE) [6, 9, $16,17]$. When originally developed by Smith, the singular value decomposition of a matrix was treated a special case of the more general singular value expansion. A history of the early development of the SVD/SVE can be found in [20]. Our study of general linear inverse problems will proceed with the singular value expansion as our primary tool. In particular, we will use the SVE for compact operators acting on Hilbert spaces. This is the analogous concept of a matrix acting on a finite dimensional vector space.

Definition 2.2.1. (Compact operator) Let $H$ and $H_{A}$ be Hilbert spaces and let $A: H \rightarrow H_{A}$ be a linear operator. We say $A$ is compact if the image under $A$ of a bounded subset of $H$ is a precompact subset of $H_{A}$.

With the assumption of compactness, a significant amount of matrix theory can be extended to continuous operators. In particular, using the SVE we decompose an operator into orthogonal functions as we would decompose a matrix into orthogonal vectors in the SVD.

Note that we do not wish to consider the case where $A$ has only finitely many singular values, nor the case where $A$ is not compact.

Theorem 2.2.1 (Singular Value Expansion). Let $H, H_{A}$ be Hilbert spaces, and let $A: H \rightarrow H_{A}$ be a compact linear operator. Then there exists orthonormal sequences 
$\left\{\phi_{k}\right\} \subset H$ and $\left\{\psi_{k}\right\} \subset H_{A}$ and positive numbers $\sigma_{1} \geq \sigma_{2} \geq \cdots$ converging to zero, such that

$$
A=\sum_{k=1}^{\infty} \sigma_{k} \psi_{k} \otimes \phi_{k}, \quad \text { and } \quad A^{*}=\sum_{k=1}^{\infty} \sigma_{k} \phi_{k} \otimes \psi_{k} .
$$

We define $\psi_{k} \otimes \phi_{k}$ as

$$
\left(\psi_{k} \otimes \phi_{k}\right) h=\left\langle h, \phi_{k}\right\rangle_{H} \psi_{k}
$$

for all $h \in H$. Note that $A^{*}$ is also a compact linear operator and denotes the adjoint of $A$. Furthermore,

$$
A \phi_{k}=\sigma_{k} \psi_{k} \text { for all } k
$$

and

$$
A h=\sum_{k=1}^{\infty} \sigma_{k}\left\langle\phi_{k}, h\right\rangle_{H} \psi_{k} \text { for all } h \in H .
$$

Additionally, $\left\{\phi_{k}\right\}$ is a complete orthonormal set for $\mathcal{N}(A)^{\perp}$ and $\left\{\psi_{k}\right\}$ is a complete orthonormal set for $\overline{\mathcal{R}(A)}$.

Proof. See [6] or [7].

To derive the singular values analytically, we typically consider solving

$$
A^{*} A \phi=\sigma^{2} \phi
$$

This yields a family of singular function, singular value pairs $\left\{\left(\sigma_{k}, \phi_{k}\right)\right\}_{k=1}^{\infty}$. As we will see, this is not at all trivial. However, the singular values in the continuous setting give analogous insights as those in the discrete setting. In particular, the singular values of an operator can define a metric for ill-posedness. A goal of this thesis is to define and understand how this metric can quantify ill-posedness in the inversion of Green's function solutions of differential equations. 
In section 2.1.2 we defined the discrete generalized inverse for a matrix $A$ that operates on real, finite dimensional Hilbert spaces with $A: \mathbb{R}^{n} \rightarrow \mathbb{R}^{m}$. Written as a summation,

$$
A^{\dagger}=\sum_{i=1}^{r} \sigma_{i}^{-1} v_{i} \otimes u_{i}=\sum_{i=1}^{r} \frac{v_{i} u_{i}^{T}}{\sigma_{i}}
$$

The solution that minimizes $\|A x-b\|_{2}^{2}$ is given by

$$
x_{\dagger}=A^{\dagger} b=\sum_{i=1}^{r} \frac{u_{i}^{T} b}{\sigma_{i}} v_{i}
$$

Now suppose $H$ and $H_{A}$ are infinite dimensional Hilbert spaces, with $A: H \rightarrow H_{A}$ a compact linear operator having singular system $\left\{\sigma_{i}, \phi_{i}, \psi_{i}\right\}$ as defined in Theorem 2.2.1. The generalized inverse is expressed in an analogous manner,

$$
A^{\dagger}=\sum_{k=1}^{\infty} \sigma_{k}^{-1} \phi_{k} \otimes \psi_{k}
$$

It is clear from this formulation that $A^{\dagger}$ is not a compact operator, since $\sigma_{k}^{-1}$ increase in an unbounded manner $[13,16]$.

As with the discrete case, it will most likely be the case that we cannot solve $A h=f$ exactly. we again find the least squares solution that minimizes $\|A h-f\|_{H_{A}}^{2}$. In terms of the SVE, this gives,

$$
h=A^{\dagger} f=\sum_{k=1}^{\infty} \sigma_{k}^{-1}\left(\phi_{k} \otimes \psi_{k}\right) f=\sum_{k=1}^{\infty} \frac{\left\langle\psi_{k}, f\right\rangle_{H_{A}}}{\sigma_{k}} \phi_{k} \text { for all } f \in D\left(A^{\dagger}\right)
$$

\subsubsection{Conditioning and ill-posedness}

The condition number of compact linear operators is still defined as $\sigma_{1} / \sigma_{r}$, however in infinite dimensions the operator can have an infinite sequence of singular values 
decaying towards zero. The condition number is therefore not a sufficient metric by which to measure ill-posedness. As in the discrete case, it is clear that small singular values (relative to $\sigma_{1}$ ) will disproportionately amplify the contribution from corresponding singular vectors or functions. If there is noise in the data, this too will be amplified, perhaps to an unacceptable level.

We characterize the ill-posedness of the problem in terms of the decay rate of its singular values. In particular, if $\sigma_{k}(A)$ decays like $k^{-q}$, we call $q$ the degree of illposedness. Thus larger values of $q$ indicate larger degrees of ill-posedness. In Chapter 3, we will use this metric to determine how joint inversion improves single inversions. Let us consider the following example to illustrate this measure of ill-posedness.

\section{Example}

Define the compact linear operator $A: H \rightarrow H_{A}$, where $H=H_{A}=L^{2}(0,1)$, by

$$
A h(t)=\int_{0}^{t} h(s) d s
$$

Then, the adjoint operator $A^{*}: H_{A} \rightarrow H$ is

$$
A^{*} f(t)=\int_{t}^{1} f(s) d s
$$

and the self-adjoint operator $A^{*} A: H \rightarrow H$ is

$$
A^{*} A h(t)=\int_{t}^{1}\left(\int_{0}^{s} h(\tau) d \tau\right) d s .
$$

Now we solve $A^{*} A \phi=\sigma^{2} \phi$ for $\sigma$ the singular values and $\phi$ the right-singular functions of $A$. If we let $\lambda=\sigma^{2}$, we may write the problem as 


$$
A^{*} A \phi=\lambda \phi(t)=\int_{t}^{1}\left(\int_{0}^{s} \phi(\tau) d \tau\right) d s .
$$

Notice, that substituting in $t=1$ to both sides of the integral equation gives us $\phi(1)=0$ for $\lambda \neq 0$. Differentiating both sides of the equation with respect to $t$ and applying a Leibniz integral rule yields

$$
\begin{aligned}
\lambda \phi^{\prime}(t) & =\frac{d}{d t} \int_{t}^{1}\left(\int_{0}^{s} \phi(\tau) d \tau\right) d s \\
& =\int_{t}^{1}\left(\frac{\partial}{\partial t} \int_{0}^{s} \phi(\tau) d \tau\right) d s-\int_{0}^{t} \phi(\tau) d \tau \\
& =-\int_{0}^{t} \phi(\tau) d \tau
\end{aligned}
$$

Substituting in $t=0$ to both sides of the above equation gives us $\phi^{\prime}(0)=0$ for $\lambda \neq 0$. Differentiating both sides of the equation again with respect to $t$ yields

$$
\begin{aligned}
\lambda \phi^{\prime \prime}(t) & =-\frac{d}{d t} \int_{0}^{t} \phi(\tau) d \tau \\
& =-\phi(t) .
\end{aligned}
$$

We can now observe that the original problem is equivalent to

$$
\lambda \phi^{\prime \prime}+\phi=0, \quad \text { with } \quad \phi(1)=\phi^{\prime}(0)=0 .
$$

To find the solution of this boundary value problem, consider the roots of the auxiliary equation $\lambda r^{2}+1=0$. They are

$$
r_{1}=\frac{1 i}{\sqrt{\lambda}}, \quad r_{2}=\frac{-1 i}{\sqrt{\lambda}}
$$


This gives us the general solution to the ODE and its derivative as

$$
\begin{aligned}
& \phi(t)=c_{1} \cos \left(\frac{t}{\sqrt{\lambda}}\right)+c_{2} \sin \left(\frac{t}{\sqrt{\lambda}}\right), \\
& \phi^{\prime}(t)=-\frac{c_{1}}{\sqrt{\lambda}} \sin \left(\frac{t}{\sqrt{\lambda}}\right)+\frac{c_{2}}{\sqrt{\lambda}} \cos \left(\frac{t}{\sqrt{\lambda}}\right) .
\end{aligned}
$$

Using the boundary conditions $\phi(1)=\phi^{\prime}(0)=0$, we deduce that

$$
\begin{aligned}
c_{2} & =0, \text { and } \\
\lambda_{k} & =\frac{4}{(2 k-1)^{2} \pi^{2}} .
\end{aligned}
$$

Thus the singular functions are of the form

$$
\phi_{k}(t)=c_{1} \cos \frac{2 k-1}{2} \pi t
$$

where $c_{1}$ must be chosen to satisfy the orthonormality condition $\left\langle\phi_{k}, \phi_{k}\right\rangle_{H}=1$, for $k=1,2, \ldots, \infty$. This yields a family of functions,

$$
\phi_{k}(t)=\sqrt{2} \cos \frac{2 k-1}{2} \pi t, k \in \mathbb{N} \text {, and } \lambda_{k}=\frac{4}{(2 k-1)^{2} \pi^{2}}, k \in \mathbb{N} .
$$

The singular values of the compact operator $A$ are the square roots of $\lambda$, that is

$$
\sigma_{k}=\frac{2}{(2 k-1) \pi}, k \in \mathbb{N}
$$

We can see from Figure 2.1 that the singular values of the operator $A$ decay like $k^{-1}$. This is classified as a mildly ill-posed problem [1]. Alternatively, the problem would be severely ill-posed if the singular values decayed at an exponential rate. The 


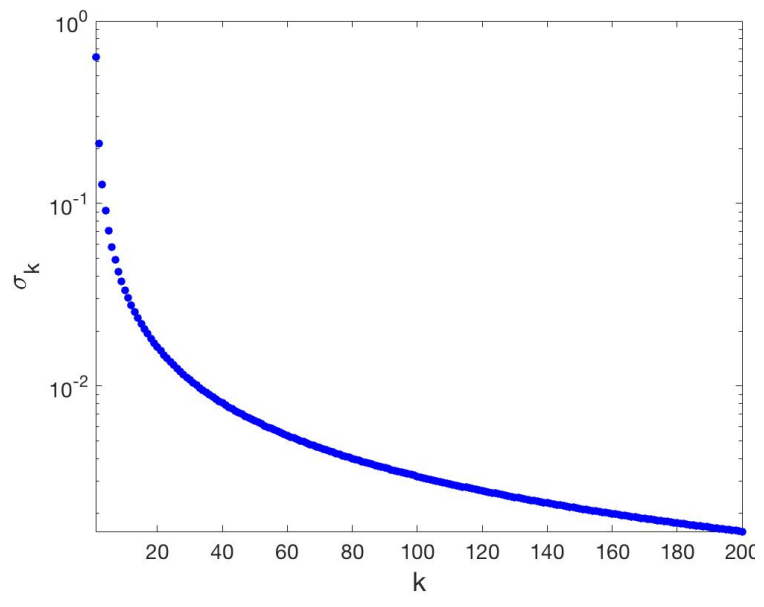

(a)

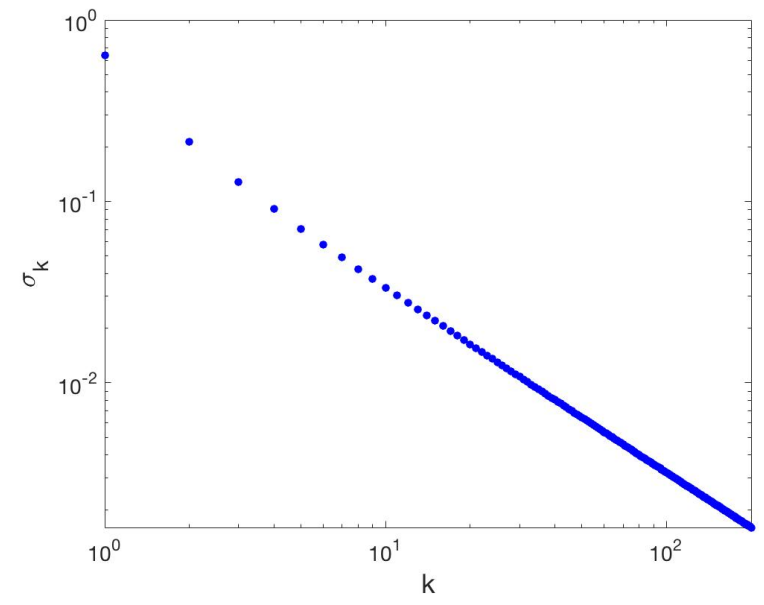

(b)

Figure 2.1: Singular values of $A$ on a semi-log scale (a), and a log-log scale (b)

diffusion equation is an example of a severely ill-posed problem.

\subsubsection{Regularization}

The negative effect decaying singular values have on the parameter estimates in an ill-posed problem can be alleviated with regularization. In infinite dimensional Hilbert spaces, a truncated SVE approximation to the operator $A$ can be formed analogously to the truncated SVD of a matrix. This requires the truncation of infinitely many singular values, and we will not investigate this finite sum approximation. Alternatively, we focus on Tikhonov regularization for compact operators.

\section{Tikhonov regularization}

The operators of interest can be expressed as an infinite summation and often do not have a well-defined inverse. Similar to the discrete case, Tikhonov regularization changes the problem to one which has an invertible operator, and therefore welldefined inverse solution. The definition of this new, invertible operator will require us 
to consider the space $H_{A} \times H=\left\{\left(h_{A}, h\right): h_{A} \in H_{A}, h \in H\right\}$ which is a Hilbert space under the inner product

$$
\left\langle\left(h_{A, 1}, h_{1}\right),\left(h_{A, 2}, h_{2}\right)\right\rangle_{H_{A} \times H}=\left\langle h_{A, 1}, h_{A, 2}\right\rangle_{H_{A}}+\left\langle h_{1}, h_{2}\right\rangle_{H} .
$$

Now we may define a new operator $T_{\lambda}: H \rightarrow H_{A} \times H$ by

$$
T_{\lambda} h=(A h, \sqrt{\lambda} h)
$$

In place of $\|A h-f\|_{H_{A}}^{2}$, we instead consider minimizing

$$
\left\|T_{\lambda} h-(f, 0)\right\|_{H_{A} \times H}^{2}=\|A h-f\|_{H_{A}}^{2}+\lambda\|h\|_{H}^{2} .
$$

Note that $\lambda$ is being used as a regularization parameter, and not an eigenvalue.

Theorem 2.2.2. Suppose $\lambda>0$. Then $\mathcal{R}\left(T_{\lambda}\right)$ is closed and $\mathcal{N}\left(T_{\lambda}\right)$ is trivial. Therefore $T_{\lambda} h=(f, 0)$ has a unique least squares solution for all $f \in H_{A},[6]$.

Proof. Consider the normal equation for this problem:

$$
\begin{aligned}
T^{*} T h & =T^{*}(f, 0) \\
T^{*}(A h, \sqrt{\lambda} h) & =T^{*}(f, 0) \\
A^{*} A h+\lambda h & =A^{*} f+\sqrt{\lambda} \cdot 0 \\
\left(A^{*} A+\lambda I\right) h & =A^{*} f .
\end{aligned}
$$

It is straightforward to show that $\left(A^{*} A+\lambda I\right)$ is invertible with a bounded inverse. Therefore a unique solution to the normal equations exists. 
Tikhonov regularization replaces the not necessarily invertible operator $A^{*} A$ with the necessarily invertible $\left(A^{*} A+\lambda I\right)$ in the normal equations. A solution is guaranteed for appropriate $\lambda$, however we have restricted the space of acceptable solutions.

We now examine the regularized solutions expressed in terms of the singular value expansion of $A$. Given the singular value expansion of $A$ and its adjoint $A^{*}, A^{*} A$ applied to $h$ can be expressed as

$$
\begin{aligned}
A^{*} A h & =A^{*}\left(\sum_{k=1}^{\infty} \sigma_{k}\left\langle\phi_{k}, h\right\rangle_{H} \psi_{k}\right) \\
& =\sum_{k=1}^{\infty} \sigma_{k}\left\langle\phi_{k}, h\right\rangle_{H} A^{*} \psi_{k} \\
& =\sum_{k=1}^{\infty} \sigma_{k}^{2}\left\langle\phi_{k}, h\right\rangle_{H} \phi_{k} .
\end{aligned}
$$

Equivalently,

$$
A^{*} A=\sum_{k=1}^{\infty} \sigma_{k}^{2} \phi_{k} \otimes \phi_{k} .
$$

Recall that $\left\{\phi_{k}\right\}$ is an orthonormal set for the $\mathcal{N}(A)^{\perp}$. So, $h \in H$ can be expressed as

$$
\begin{aligned}
h & =\operatorname{proj}_{\mathcal{N}(A)^{\perp}} h+\operatorname{proj}_{\mathcal{N}(A)} h \\
& =\sum_{k=1}^{\infty}\left\langle h, \phi_{k}\right\rangle_{H} \phi_{k}+\operatorname{proj}_{\mathcal{N}(A)} h
\end{aligned}
$$

We may now consider writing the Tikhonov operator $\left(A^{*} A+\lambda I\right)$ applied to $h$ in terms of the SVE of $A$. Namely, 


$$
\begin{aligned}
\left(A^{*} A+\lambda I\right) h & =A^{*} A\left(\sum_{k=1}^{\infty}\left\langle h, \phi_{k}\right\rangle_{H} \phi_{k}+\operatorname{proj}_{\mathcal{N}(A)} h\right)+\lambda\left(\sum_{k=1}^{\infty}\left\langle h, \phi_{k}\right\rangle_{H} \phi_{k}+\operatorname{proj}_{\mathcal{N}(A)} h\right) \\
& =A^{*} A\left(\sum_{k=1}^{\infty}\left\langle h, \phi_{k}\right\rangle_{H} \phi_{k}\right)+\lambda \sum_{k=1}^{\infty}\left\langle h, \phi_{k}\right\rangle_{H} \phi_{k}+\lambda \operatorname{proj}_{\mathcal{N}(A)} h \\
& =\sum_{k=1}^{\infty}\left\langle h, \phi_{k}\right\rangle_{H} A^{*} A \phi_{k}+\sum_{k=1}^{\infty} \lambda\left\langle h, \phi_{k}\right\rangle_{H} \phi_{k}+\lambda \operatorname{proj}_{\mathcal{N}(A)} h \\
& =\sum_{k=1}^{\infty} \sigma_{k}^{2}\left\langle h, \phi_{k}\right\rangle_{H} \phi_{k}+\sum_{k=1}^{\infty} \lambda\left\langle h, \phi_{k}\right\rangle_{H} \phi_{k}+\lambda \operatorname{proj}_{\mathcal{N}(A)} h \\
& =\sum_{k=1}^{\infty}\left(\sigma_{k}^{2}+\lambda\right)\left\langle h, \phi_{k}\right\rangle_{H} \phi_{k}+\lambda \operatorname{proj}_{\mathcal{N}(A)} h .
\end{aligned}
$$

Equivalently,

$$
\left(A^{*} A+\lambda I\right)=\sum_{k=1}^{\infty}\left(\sigma_{k}^{2}+\lambda\right) \phi_{k} \otimes \phi_{k}+\lambda \operatorname{proj}_{\mathcal{N}(A)}
$$

This allows us to consider solutions $h^{(\lambda)} \in H$ satisfying $\left(A^{*} A+\lambda I\right) h^{(\lambda)}=A^{*} f$ in terms of the SVE of $A$. From our above expansions, we can see that $h^{(\lambda)}$ must equivalently satisfy

$$
\sum_{k=1}^{\infty}\left(\sigma_{k}^{2}+\lambda\right)\left\langle h^{(\lambda)}, \phi_{k}\right\rangle_{H} \phi_{k}+\lambda \operatorname{proj}_{\mathcal{N}(A)} h^{(\lambda)}=\sum_{k=1}^{\infty} \sigma_{k}\left\langle\psi_{k}, f\right\rangle_{H_{A}} \phi_{k}
$$

Since the two summations are linear combinations of $\left\{\phi_{k}\right\}$, they represent elements in $\mathcal{N}(A)^{\perp}$. Therefore, it must be the case that $\lambda \operatorname{proj}_{\mathcal{N}(A)} h^{(\lambda)}=0$, and $h^{(\lambda)}$ lies in $\mathcal{N}(A)^{\perp}$. So we have that $h^{(\lambda)}$ must satisfy

$$
\sum_{k=1}^{\infty}\left(\sigma_{k}^{2}+\lambda\right)\left\langle h^{(\lambda)}, \phi_{k}\right\rangle_{H} \phi_{k}=\sum_{k=1}^{\infty} \sigma_{k}\left\langle\psi_{k}, f\right\rangle_{H_{A}} \phi_{k}
$$




$$
\begin{aligned}
& \Longrightarrow\left(\sigma_{k}^{2}+\lambda\right)\left\langle h^{(\lambda)}, \phi_{k}\right\rangle_{H}=\sigma_{k}\left\langle\psi_{k}, f\right\rangle_{H_{A}} \text { for all } k \in \mathbb{N} \\
& \Longrightarrow\left\langle h^{(\lambda)}, \phi_{k}\right\rangle_{H}=\frac{\sigma_{k}}{\sigma_{k}^{2}+\lambda}\left\langle\psi_{k}, f\right\rangle_{H_{A}} \text { for all } k \in \mathbb{N} \\
& \Longrightarrow h^{(\lambda)}=\sum_{k=1}^{\infty} \frac{\sigma_{k}}{\sigma_{k}^{2}+\lambda}\left\langle\psi_{k}, f\right\rangle_{H_{A}} \phi_{k} .
\end{aligned}
$$

This implies that the generalized inverse operator for the modified least squares problem is

$$
A_{\lambda}^{\dagger}=\left(A^{*} A+\lambda I\right)^{-1} A^{*}=\sum_{k=1}^{\infty} \frac{\sigma_{k}}{\sigma_{k}^{2}+\lambda} \phi_{k} \otimes \psi_{k}
$$

Notice that

$$
\frac{\sigma_{k}}{\sigma_{k}^{2}+\lambda} \rightarrow 0, \text { as } k \rightarrow \infty
$$

Therefore the operator $A_{\lambda}^{\dagger}$ is bounded, and inverse solutions depend continuously on $f$.

These solution estimates depend strongly on the regularization parameter $\lambda$, which restricts the space of acceptable solutions. In this thesis we study the impact of using physics and corresponding observations to restrict the solution space. This will allow more physically relevant solutions and is typically called simultaneous joint inversion. Joint inversion restricts the parameters to ones that satisfy two or more models. Joint inversion may not necessarily result in a well posed problem, but should require less regularization. 


\section{CHAPTER 3}

\section{JOINT INVERSION}

\subsection{Discrete joint inversion}

\subsubsection{Linear problems}

The earliest mention of joint inversion comes from Vozoff and Jupp in their 1975 paper [22]. Here the authors considered combining several different kinds of geophysical measurements to avoid some of the ambiguity inherent in the individual methods. Mathematically speaking, they looked at a problem of two linear models $A$ and $B$ dependent on the same set of unknown parameters $x$, with corresponding data $d_{1}$ and $d_{2}$ respectively:

$$
A x=d_{1} \text { and } B x=d_{2} .
$$

When $A$ and $B$ are ill-conditioned, the individual problems require regularization. Alternatively, Vozoff and Jupp explored the potential of using less regularization by solving the joint inversion problem

$$
\left[\begin{array}{l}
A \\
B
\end{array}\right] x=\left[\begin{array}{l}
d_{1} \\
d_{2}
\end{array}\right] .
$$


To measure the effectiveness of this approach, the authors considered the decay rate of the singular values of $A, B$, and $\left[\begin{array}{l}A \\ B\end{array}\right]$. In several examples, they demonstrated that the singular value decomposition of $\left[\begin{array}{l}A \\ B\end{array}\right]$ yielded a significant increase in the number of usable values, as compared to $A$ and $B$ individually.

In this thesis we extend the discrete results of Vozoff and Jupp to a continuous setting. In particular, we measure the effectiveness of combining operators in an analogous way; the decay rate of singular values from individual and joint compact operators will be compared.

\subsubsection{Green's functions example}

First, we apply the ideas presented by Vozoff and Jupp to a pair of discretized Green's functions. As motivated by the imaging example in the introduction, we choose the Green's functions for the wave and diffusion equations in time and one spatial variable.

The Green's function for the wave and diffusion equations for homogeneous media in $x$ and $t$ are

$$
\begin{aligned}
K_{w} & =\frac{1}{2} \mathcal{H}((t-\tau)+(x-\xi)) \mathcal{H}((t-\tau)-(x-\xi)), \\
K_{d} & =\frac{\mathcal{H}(t-\tau)}{\sqrt{4 \pi^{2}(t-\tau)}} \exp \left(\frac{-(x-\xi)^{2}}{4(t-\tau)}\right)
\end{aligned}
$$

respectively, where $\mathcal{H}$ denotes the Heaviside function [3].

Given each kernel $K$, a solution $u$ to the corresponding differential equation with source term $f$ can be expressed as 


$$
u(x, t)=\iint K(x, t, \xi, \tau) f(\xi, \tau) d \xi d \tau
$$

Using a two dimensional midpoint quadrature rule, we can approximate our solution on an $m \times n$ grid as

$$
u\left(x_{i}, t_{j}\right) \approx \sum_{l=1}^{m} \sum_{k=1}^{n} K\left(x_{i}, t_{j}, \xi_{k}, \tau_{l}\right) f\left(\xi_{k}, \tau_{l}\right) \Delta \xi \Delta \tau
$$

With some simple reshaping, we approximate the operation of double integration against $K$ as a matrix multiplication so that we solve $A f=u$ for the source function. The resulting singular values of the matrices from the discretized individual and joint operators are plotted in Figure 3.1. The wave equation in (a) had singular values decay slowly until the ninetieth. Then the singular values rapidly approached zero. The diffusion equation in (b) had singular values that decayed more rapidly than the wave equation, exhibiting noticeable drops every ten values past the forty-fifth, eventually dropping to machine precision. The joint operator in (c) had singular values that tapered off far less dramatically than either the wave or diffusion equation. It is significantly better conditioned than either individual model.

It must be noted that even though this simple discretization resulted in improved decay rates of singular values in joint inversion this will not always be the case. Having run many numerical experiments, it is still unclear what underlying features cause discrete models to interact well in a joint inversion. For this reason, we explore the more general notion of joint inversion on compact operators. In so doing, we more deeply understand what properties in physical models improve inverse estimates in ill-posed problems. 


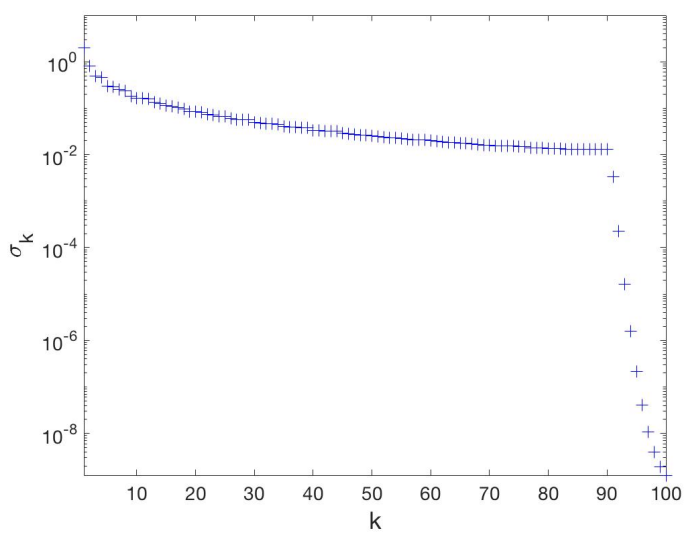

(a) $\sigma\left(K_{w}\right)$

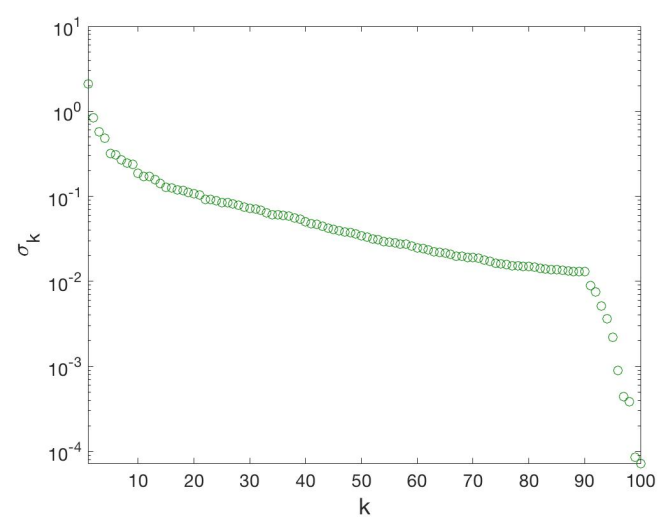

(c) $\sigma\left(K_{w, d}\right)$

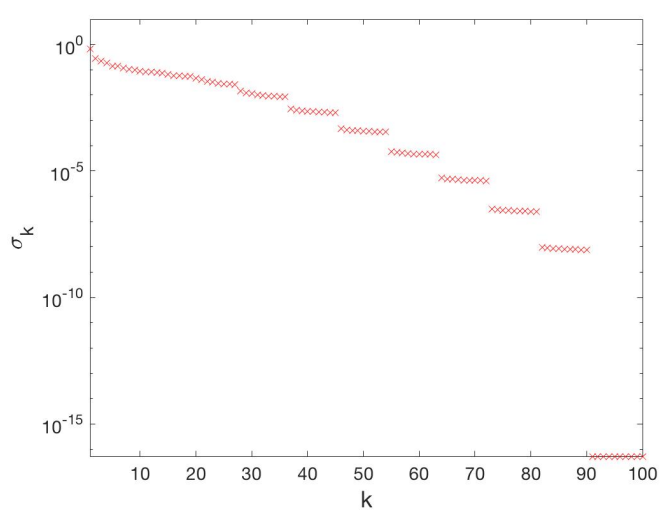

(b) $\sigma\left(K_{d}\right)$

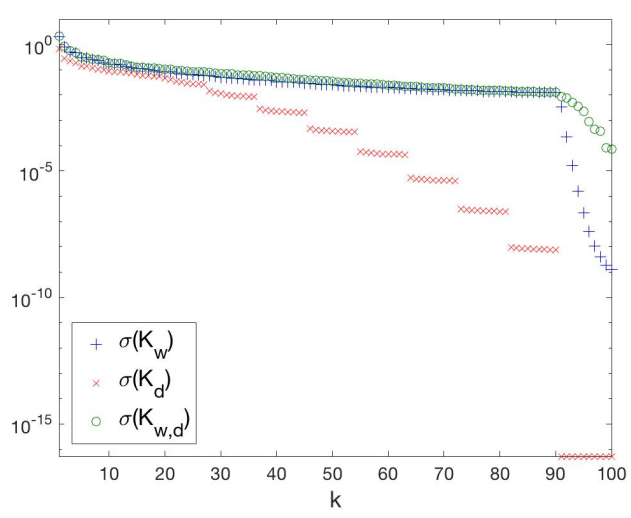

(d) overlayed

Figure 3.1: Singular values of individual operators and joint operator

\subsection{Joint inversion with compact operators}

Vozoff and Jupp vertically concatenated two discrete linear models into one stacked matrix. We now define a joint operator that combines two compact operators in an analogous way. The decay rate of the singular values of this joint operator compared to the decay rate of the singular values of the individual operators will give an indication of the effectiveness of joint inversion. 


\subsubsection{Joint operators}

As with Tikhonov regularization, our joint operator will map into the Cartesian product of two Hilbert spaces. However, rather than consider the mathematically defined space $H$ in Tikhonov regularization, we introduce the new physical space $H_{B}$ defined by an additional data collection technique.

Definition 3.2.1. (Hilbert space direct sum) Let $H_{A}$ and $H_{B}$ be Hilbert spaces and

$$
H_{A} \oplus H_{B}=\left\{\left(h_{A}, h_{B}\right): h_{A} \in H_{A}, h_{B} \in H_{B}\right\}
$$

Define an inner product $\langle\cdot, \cdot\rangle$ on $H_{A} \oplus H_{B}$ by

$$
\left\langle\left(h_{A, 1}, h_{B, 1}\right),\left(h_{A, 2}, h_{B, 2}\right)\right\rangle_{H_{A} \oplus H_{B}}=\left\langle h_{A, 1}, h_{A, 2}\right\rangle_{H_{A}}+\left\langle h_{B, 1}, h_{B, 2}\right\rangle_{H_{B}}
$$

With respect to this inner product, $H_{A} \oplus H_{B}$ is a Hilbert Space called the Hilbert space direct sum of $H_{A}$ and $H_{B}$.

Remark. Let $A: H \rightarrow H_{A}$ and $B: H \rightarrow H_{B}$ be compact operators from the Hilbert space $H$ to the Hilbert spaces $H_{A}$ and $H_{B}$ respectively. Define $C: H \rightarrow H_{A} \oplus H_{B}$ as $C h=(A h, B h)$, for all $h \in H$. Then $C$ is a compact operator between two Hilbert spaces, [2]. Therefore, $C$ admits a singular value expansion.

As mentioned previously, the decay rate of the singular values provide a metric for the ill-posedness of an operator. With $A, B$, and $C$ defined as above, we will compare the singular value decay rates of the three operators to see if the joint operator yielded any improvement. For the purposes of visualization, it is helpful to think of $C$ as defining a parametric curve in the space $H_{A} \oplus H_{B}$. Then it is clear that if $A$ and $B$ are compact, so is $C$. 


\section{Example}

Define the Hilbert spaces $H=L^{2}(0,2 \pi)$, and $H_{A}=H_{B}=\mathbb{R}$. Define the compact operators $A: H \rightarrow H_{A}$ and $B: H \rightarrow H_{B}$ as

$$
A h=\int_{0}^{2 \pi} h(y) \delta(y-5) d y, \quad B h=\int_{0}^{2 \pi} h(y) \delta(y-7) d y
$$

Define $C: H \rightarrow H_{A} \oplus H_{B}$ as

$$
C h=(A h, B h)=\left(\int_{0}^{2 \pi} h(y) \delta(y-5) d y, \int_{0}^{2 \pi} h(y) \delta(y-7) d y\right)
$$

Now let us consider the image of a subset of $H$ under $C$. Define $S=\{\cos k x: k \in \mathbb{R}, x \in[0,2 \pi]\}$, a continuum of cosine functions of each possible frequency. So for each frequency $k \in \mathbb{R}$ the mapping of $\cos k x$ under $C$ is

$$
\begin{aligned}
C(\cos k x) & =\left(\int_{0}^{2 \pi} \cos (k y) \delta(y-5) d y, \int_{0}^{2 \pi} \cos (k y) \delta(y-7) d y\right) \\
& =(\cos (k \cdot 5), \cos (k \cdot 7)) .
\end{aligned}
$$

Since the codomain of $C$ is $H_{A} \oplus H_{B}=\mathbb{R}^{2}$, we can represent the image of $S$ under $C$ graphically; see Figure 3.2.

\subsubsection{Singular values}

The singular values of the joint operator are found by solving $C^{*} C \phi=\sigma^{2} \phi$. With $C$ defined as in the remark above, $h_{A} \in H_{A}$ and $h_{B} \in H_{B}$, the adjoint $C^{*}: H_{A} \oplus H_{B} \rightarrow$ $H$ is

$$
C^{*}\left(h_{A}, h_{B}\right)=A^{*} h_{A}+B^{*} h_{B} .
$$




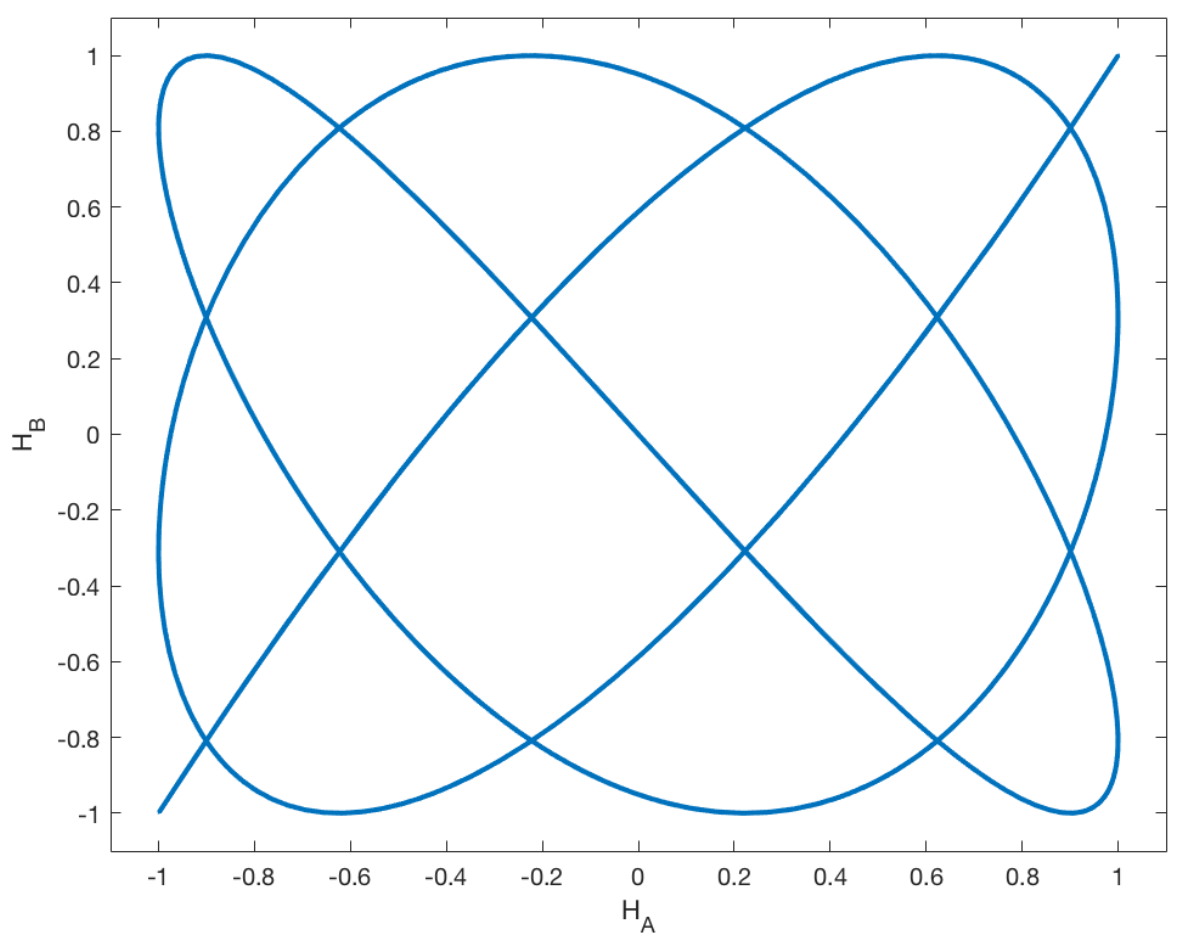

Figure 3.2: Parametric curve defined by $C(\cos k x)$ for $k \in[-5,5]$

Expanding we get

$$
\begin{aligned}
\sigma^{2} \phi & =C^{*} C \phi \\
& =C^{*}(A \phi, B \phi) \\
& =A^{*} A \phi+B^{*} B \phi .
\end{aligned}
$$

It is a challenge to solve this equation for the singular values and functions analytically. In the case where $A$ and $B$ are Green's function operators associated with the differential operations $L_{A}$ and $L_{B}$ respectively, we can see that this is an integral equation. For our motivating imaging problem, 


$$
A h=\int_{\Omega} K_{w} h \quad \text { and, } \quad B h=\int_{\Omega} K_{d} h
$$

where $h \in H$, and $K_{w}, K_{d}$ are the Green's functions solutions to the wave and diffusion equations respectively. Since we are better equipped to solve differential equations, we take steps to transform equation (3.3) into an equivalent ODE. The following properties are what allows us to do so.

Remark. Let $\mathcal{L}$ be a linear differential operator, and $K$ be the corresponding Green's function which satisfies $\mathcal{L} K(t, s)=\delta(t-s)$. Let $A$ be defined as $A h=\int_{\Omega} K(t, s) h(s) d s$ for all $h$ in the relevant Hilbert space $H$. Then we have

$$
\begin{aligned}
\int_{\Omega} \mathcal{L} K(t, s) h(s) d s & =\int_{\Omega} \delta(t-s) h(s) d s \\
\mathcal{L} \int_{\Omega} K(t, s) h(s) d s & =h(t) \\
\mathcal{L} A h(t) & =h(t),
\end{aligned}
$$

for all $h \in H$. Similarly we have,

$$
\mathcal{L}^{*} A^{*} h_{A}(t)=h_{A}(t)
$$

for all $h_{A}$ in the relevant codomain $H_{A}$.

We begin the process of transforming equation (3.3) by applying the adjoint differential operator, $L_{A}^{*}$ to both sides and simplifying using the property in equation $(3.5)$ : 


$$
\begin{aligned}
L_{A}^{*}\left(\sigma^{2} \phi\right) & =L_{A}^{*}\left(A^{*} A \phi+B^{*} B \phi\right), \\
\sigma^{2} L_{A}^{*} \phi & =A \phi+L_{A}^{*} B^{*} B \phi .
\end{aligned}
$$

Now we apply the differential operator $L_{A}$ to both sides and simplify using the property in equation (3.4):

$$
\begin{aligned}
L_{A}\left(\sigma^{2} L_{A}^{*} \phi\right) & =L_{A}\left(A \phi+L_{A}^{*} B^{*} B \phi\right), \\
\sigma^{2} L_{A} L_{A}^{*} \phi & =\phi+L_{A} L_{A}^{*} B^{*} B \phi .
\end{aligned}
$$

This eliminated the integrals associated with the operator $A^{*}$ and $A$. We must also apply $L_{B}^{*}$ and $L_{B}$ in the same way to eliminate the integrals associated with $B^{*}$ and B.

Certain linear differential operators commute, for instance, linear differential operators in one dimension, with constant coefficients. Below we use the assumption of commutativity so that we can define an ODE from which we can find the singular values of the joint operator. It is not a realistic assumption for the application that motivates this work. However, we view this as preliminary work that can be extended to more realistic settings.

Assuming $L_{A} L_{A}^{*}$ and $L_{B} L_{B}^{*}$ commute and applying $L_{B} L_{B}^{*}$ to both sides of the equation, we find 


$$
\begin{aligned}
& L_{B} L_{B}^{*}\left(\sigma^{2} L_{A} L_{A}^{*} \phi\right)=L_{B} L_{B}^{*}\left(\phi+L_{A} L_{A}^{*} B^{*} B \phi\right) \\
& \sigma^{2}\left(L_{B} L_{B}^{*} L_{A} L_{A}^{*}\right) \phi=L_{B} L_{B}^{*} \phi+L_{A} L_{A}^{*} L_{B} L_{B}^{*} B^{*} B \phi \\
& \sigma^{2}\left(L_{B} L_{B}^{*} L_{A} L_{A}^{*}\right) \phi=L_{B} L_{B}^{*} \phi+L_{A} L_{A}^{*} \phi \\
& \sigma^{2}\left(L_{B} L_{B}^{*} L_{A} L_{A}^{*}\right) \phi=\left(L_{B} L_{B}^{*}+L_{A} L_{A}^{*}\right) \phi .
\end{aligned}
$$

Though not necessarily aesthetically pleasing, this is clearly an ODE in $\phi$. Namely,

$$
\sigma^{2}\left(L_{B} L_{B}^{*} L_{A} L_{A}^{*}\right) \phi=\left(L_{B} L_{B}^{*}+L_{A} L_{A}^{*}\right) \phi
$$

This produced an ODE with much higher order than that of the given differential operators $L_{A}$ or $L_{B}$, and introduced many more boundary conditions. In cases where equation (3.6) is too difficult to solve, we turn to a numerical approximation as described in Section 3.2.3.

\subsubsection{Approximation of SVE with SVD}

The singular value expansion of an individual integral kernel, such as a Green's function, can be approximated using the Galerkin method. It has been shown that the singular values derived using the Galerkin method converge to the true singular values. For further details concerning the results pertaining to individual operators, see $[9,18]$. Here, we describe the algorithm for computing the SVE approximation and its convergence properties. We then extend the methods to joint operators, and prove the corresponding convergence properties.

Given a Green's function or other integral kernel, $K_{A}(s, t)$ defined over $\Omega_{s} \times \Omega_{t}$ and associated integral operator $A$, we choose orthonormal bases $\left\{p_{j}(t)\right\}_{j=1}^{n}$ and $\left\{q_{i}(s)\right\}_{i=1}^{n}$

for $L^{2}\left(\Omega_{t}\right)$ and $L^{2}\left(\Omega_{s}\right)$ respectively. The matrix $A^{(n)}$ with entries $a_{i j}^{(n)}$ approximates 
the operator $A$, and is defined by

$$
\begin{aligned}
a_{i j}^{(n)} & =\left\langle q_{i}, A p_{j}\right\rangle \\
& =\left\langle q_{i},\left\langle K_{A}, p_{j}\right\rangle\right\rangle \\
& =\int_{\Omega_{s}} \int_{\Omega_{t}} q_{i}(s) K_{A}(s, t) p_{j}(t) d t d s .
\end{aligned}
$$

The SVD $A^{(n)}$ is denoted $U^{(n)} \Sigma^{(n)}\left(V^{(n)}\right)^{T}$ with $\Sigma^{(n)}=\operatorname{diag}\left(\sigma_{1}^{(n)}, \sigma_{2}^{(n)}, \ldots \sigma_{n}^{(n)}\right)$ containing the discrete singular values $\sigma_{i}^{(n)}$ which approximate the continuous singular values $\sigma_{i}$.

Theorem 3.2.1. (SVE-SVD, [9, 18]) Define

$$
\begin{aligned}
\left(\Delta_{A}^{(n)}\right)^{2} & =\left\|K_{A}\right\|^{2}-\left\|A^{(n)}\right\|_{F}^{2} \\
& =\sum_{i=1}^{\infty} \sigma_{i}^{2}-\sum_{i=1}^{n}\left(\sigma_{i}^{(n)}\right)^{2}
\end{aligned}
$$

Then the following hold for all $i$ and $n$, independent of the convergence of $\Delta_{A}^{(n)}$ to 0 :

1. $\sigma_{i}^{(n)} \leq \sigma_{i}^{(n+1)} \leq \sigma_{i}$

2. $0 \leq \sigma_{i}-\sigma_{i}^{(n)} \leq \Delta_{A}^{(n)}$

Thus if $\lim _{n \rightarrow \infty}\left(\Delta_{A}^{(n)}\right)^{2}=0$, the singular values $\sigma_{i}$ of $K_{A}$ are accurately approximated.

We extend this method to joint operators in the following fashion. Let $K_{A}(s, t)$ and $K_{B}(s, t)$ be Green's functions or other integral kernels, over $\Omega_{s} \times \Omega_{t}$ with associated integral operator $A$ and $B$ respectively, and $K_{A} \oplus K_{B}$ is the kernel of the direct sum integral operator $C=A \oplus B,[8]$. Compute $A^{(n)}$ and $B^{(n)}$ separately as described in equation (3.7). Now that we are in a discrete setting we can stack these matrices 
found to form $C^{(n)}=\left[\begin{array}{l}A^{(n)} \\ B^{(n)}\end{array}\right]$. We now show that the singular values of this matrix $\sigma_{i}\left(C^{(n)}\right)$ converge to the singular values $\sigma_{i}(C)$ of the operator $C$. We will begin by using an equivalent definition for the singular values and a Lemma which can be found in $[9]$.

Definition 3.2.2. The singular values of an integral operator $A$ with a real, square integrable kernel $K_{A}$ are the stationary values of the functional

$$
F[\phi, \psi]=\frac{\langle\psi, A \phi\rangle}{\|\phi\|\|\psi\|}
$$

with the corresponding left and right singular functions given by $\phi /\|\phi\|$ and $\psi /\|\psi\|$ respectively.

The idea is then to approximate $A$ with an integral operator whose kernel is degenerate. We accomplish this by restricting $\phi$ and $\psi$ to a the span of finitely many, $n$, orthonormal basis functions.

Theorem 3.2.2 ([9]). The approximate singular values $\sigma_{i}\left(C^{(n)}\right)$, where $n$ is the number of basis functions, are increasingly better approximations to the true singular values $\sigma_{i}(C)$

$$
\sigma_{i}\left(C^{(n)}\right) \leq \sigma_{i}\left(C^{(n+1)}\right) \leq \sigma_{i}(C), \quad i=1,2, \ldots n
$$

Proof. The theorem follows from the facts that the basis functions $\left\{\phi_{i}\right\}_{i=1}^{n}$ and $\left\{\psi_{i}\right\}_{i=1}^{n}$ are orthonormal and that the singular values $\sigma_{i}\left(C^{(n)}\right)$ and $\sigma_{i}\left(C^{(n+1)}\right)$ are the stationary values of $F[\phi, \psi]$ with $\phi$ and $\psi$ restricted to $n$-dimensional and $n+1$-dimensional function subspaces respectively.

Now we consider the error of joint approximation. 
Theorem 3.2.3. Define

$$
\begin{aligned}
\left(\Delta_{C}^{(n)}\right)^{2} & =\left\|K_{A} \oplus K_{B}\right\|^{2}-\left\|\left[\begin{array}{l}
A^{(n)} \\
B^{(n)}
\end{array}\right]\right\|_{F}^{2} \\
& =\sum_{i=1}^{\infty} \sigma_{i}(C)^{2}-\sum_{i=1}^{n} \sigma_{i}\left(C^{(n)}\right)^{2}
\end{aligned}
$$

$\left(\Delta_{C}^{(n)}\right)^{2}$ is equivalent to $\left(\Delta_{A}^{(n)}\right)^{2}+\left(\Delta_{B}^{(n)}\right)^{2}$, the sum of the errors from the discretization of $A$ and $B$.

Proof. Expanding equation (3.8),

$$
\begin{aligned}
\left(\Delta_{C}^{(n)}\right)^{2} & =\left\langle K_{A} \oplus K_{B}, K_{A} \oplus K_{B}\right\rangle-\left[\left\|A^{(n)}\right\|_{F}^{2}+\left\|B^{(n)}\right\|_{F}^{2}\right] \\
& =\left\langle K_{A}, K_{A}\right\rangle+\left\langle K_{B}, K_{B}\right\rangle-\left\|A^{(n)}\right\|_{F}^{2}-\left\|B^{(n)}\right\|_{F}^{2} \\
& =\left\|K_{A}\right\|^{2}-\left\|A^{(n)}\right\|_{F}^{2}+\left\|K_{B}\right\|^{2}-\left\|B^{(n)}\right\|_{F}^{2} \\
& =\left(\Delta_{A}^{(n)}\right)^{2}+\left(\Delta_{B}^{(n)}\right)^{2} .
\end{aligned}
$$

Thus the joint error is the sum of the individual errors.

This quantity is important in bounding the error.

Theorem 3.2.4. The sum of squares error of the approximate joint singular values is bounded by

$$
\sum_{i=1}^{n}\left[\sigma_{i}(C)-\sigma_{i}\left(C^{(n)}\right)\right]^{2} \leq\left(\Delta_{C}^{(n)}\right)^{2}
$$

\section{Proof.}




$$
\begin{aligned}
\sum_{i=1}^{n}\left[\sigma_{i}(C)-\sigma_{i}\left(C^{(n)}\right)\right]^{2} & =\sum_{i=1}^{n}\left[\sigma_{i}(C)\right]^{2}+\sum_{i=1}^{n}\left[\sigma_{i}\left(C^{(n)}\right)\right]^{2}-2 \sum_{i=1}^{n} \sigma_{i}(C) \sigma_{i}\left(C^{(n)}\right) \\
& \leq \sum_{i=1}^{n}\left[\sigma_{i}(C)\right]^{2}+\sum_{i=1}^{n}\left[\sigma_{i}\left(C^{(n)}\right)\right]^{2}-2 \sum_{i=1}^{n}\left[\sigma_{i}\left(C^{(n)}\right)\right]^{2} \\
& \leq \sum_{i=1}^{n}\left[\sigma_{i}(C)\right]^{2}+\sum_{i=1}^{n}\left[\sigma_{i}\left(C^{(n)}\right)\right]^{2} \\
& =\left(\Delta_{C}^{(n)}\right)^{2} \\
& =\left(\Delta_{A}^{(n)}\right)^{2}+\left(\Delta_{B}^{(n)}\right)^{2}
\end{aligned}
$$

Thus, if $\lim _{n \rightarrow \infty}\left(\Delta_{A}^{(n)}\right)^{2}=0$ and $\lim _{n \rightarrow \infty}\left(\Delta_{B}^{(n)}\right)^{2}=0$, the singular values $\sigma(C)$ are accurately approximated by $\sigma\left(C^{(n)}\right)$. 


\section{CHAPTER 4}

\section{APPLICATION WITH COMPACT OPERATORS}

In this chapter, we give an example of our approach to determining the effectiveness of joint inversion by calculating the decay rate of singular values. Models based on the wave and the diffusion equations give the motivation for this work, but determining the corresponding singular values is outside the scope of this thesis. Therefore we apply our approach to the one dimensional steady state versions of the models.

\subsection{Individual operators}

The Green's functions for the two differential operators

$$
\begin{aligned}
& L_{A} u=-u^{\prime \prime}, \quad u(0)=u(\pi)=0, \\
& L_{B} u=u^{\prime \prime}+b^{2} u, \quad u(0)=u(\pi)=0, \quad \text { and } b \notin \mathbb{Z}
\end{aligned}
$$

are well known, [3]. Let $A: L^{2}[0, \pi] \rightarrow L^{2}[0, \pi]$ be defined by

$$
A h(x)=\int_{0}^{\pi} K_{A}(x, y) h(y) d y
$$

where the kernel 


$$
K_{A}= \begin{cases}\frac{1}{\pi}(\pi-x) y, & 0 \leq y \leq x \leq \pi \\ \frac{1}{\pi}(\pi-y) x, & 0 \leq x \leq y \leq \pi\end{cases}
$$

Then $A f=u$ where $-u^{\prime \prime}=f$ with zero Dirichlet boundary conditions.

Similarly, let $B: L^{2}[0, \pi] \rightarrow L^{2}[0, \pi]$ be defined by

$$
B h(x)=\int_{0}^{\pi} K_{B}(x, y) h(y) d y
$$

where the kernel

$$
K_{B}= \begin{cases}-\frac{\sin (b y) \sin [b(\pi-x)]}{b \sin (b \pi)}, & 0 \leq y \leq x \leq \pi \\ -\frac{\sin (b x) \sin [b(\pi-y)]}{b \sin (b \pi)}, & 0 \leq x \leq y \leq \pi\end{cases}
$$

Then $B f=u$, where $u^{\prime \prime}+b^{2} u=f$ with zero Dirichlet boundary conditions. Thus $A$ and $B$ are the Green's function operators associated with $L_{A}$ and $L_{B}$, respectively.

$A$ is a self-adjoint compact operator, and therefore admits an eigenvalue expansion. In this case, the singular values of $A$ are simply the absolute values of the eigenvalues. The equation $A \phi=\lambda \phi$ is equivalent to

$$
\lambda \phi(x)=\int_{0}^{\pi} K_{A}(x, y) \phi(y) d y
$$

First notice that when evaluated at $x=0$ or $x=\pi$, the integral of the kernel $K_{A}$ is zero. This implies the conditions $\phi(0)=0$ and $\phi(\pi)=0$. Now, differentiating both sides of the integral equation with respect to $x$ and applying the Leibniz integral rule yields 


$$
\begin{aligned}
\lambda \phi^{\prime}(x) & =\frac{d}{d x} \int_{0}^{\pi} K_{x}(x, y) \phi(y) d y \\
& =\int_{0}^{\pi} \frac{\partial}{\partial x}[K(x, y) \phi(y)] d y \\
& =\int_{0}^{x} \frac{\partial}{\partial x}[K(x, y)] \phi(y) d y+\int_{x}^{\pi} \frac{\partial}{\partial x}[K(x, y)] \phi(y) d y \\
& =\int_{0}^{x}-\frac{1}{\pi} y \phi(y) d y+\int_{x}^{\pi} \frac{1}{\pi}(\pi-y) \phi(y) d y .
\end{aligned}
$$

Differentiating both sides with respect to $x$ and applying the Leibniz integral rule again gives

$$
\begin{aligned}
\lambda \phi^{\prime \prime}(x) & =\frac{d}{d x}\left(\int_{0}^{x}-\frac{1}{\pi} y \phi(y) d y+\int_{x}^{\pi} \frac{1}{\pi}(\pi-y) \phi(y) d y\right) \\
& =-\frac{1}{\pi} x \phi(x)-\frac{1}{\pi}(\pi-x) \phi(x) \\
& =-\phi(x) .
\end{aligned}
$$

We may now observe that the original eigenvalue problem is equivalent to the ODE

$$
\lambda \phi^{\prime \prime}=-\phi \text { with } \phi(0)=\phi(\pi)=0 \text {. }
$$

Solutions to this boundary value problem are of the form

$$
\phi(x)=c_{1} \cos \left(\frac{1}{\sqrt{\lambda}} x\right)+c_{2} \sin \left(\frac{1}{\sqrt{\lambda}} x\right)
$$

Using the boundary conditions, we deduce that 


$$
\begin{aligned}
& c_{1}=0, \quad \text { and } \\
& \lambda_{k}=\frac{1}{k^{2}}, \quad \text { for } k=1,2, \ldots, \infty .
\end{aligned}
$$

Thus solutions are of the form

$$
\phi_{k}(x)=c_{2} \sin (k x)
$$

where $c_{2}$ must be chosen to satisfy the condition $\left\langle\phi_{k}, \phi_{k}\right\rangle_{L^{2}[0, \pi]}=1$, for $k=1,2, \ldots, \infty$. This yields a family of solutions,

$$
\phi_{k}(x)=\sqrt{\frac{2}{\pi}} \sin (k x), \quad \text { and } \quad \lambda_{k}=\frac{1}{k^{2}}, \quad \text { for } k=1,2, \ldots, \infty
$$

Since $\lambda_{k}>0$, the singular values for $A$ are also $\sigma_{k}=\frac{1}{k^{2}}$ for $k=1,2, \ldots, \infty$.

$B$ is also a self-adjoint operator, so we need only compute its eigenvalues. Note that we will reuse the $\phi, \lambda$ notation, but the solutions obtained here are not related to $A$. We follow an approach similar to that for the computation of the singular values of $A$, and we exploit the relationship between $B$ and $L_{B}$.

Applying $L_{B}$ to both sides, we find

$$
\begin{gathered}
L_{B} B \phi=L_{B}(\lambda \phi) \\
\phi=\lambda L_{B} \phi \\
\Longrightarrow \phi=\lambda\left(\phi^{\prime \prime}+b^{2} \phi\right), \quad \phi(0)=\phi(\pi)=0 .
\end{gathered}
$$

So we are left with the following equations 


$$
\left(\frac{1}{\lambda}-b^{2}\right) \phi=\phi^{\prime \prime}, \quad \phi(0)=\phi(\pi)=0
$$

Solutions to this boundary value problem are of the form

$$
\phi(x)=c_{1} \cos \left(\sqrt{\frac{1}{\lambda}-b^{2}} x\right)+c_{2} \sin \left(\sqrt{\frac{1}{\lambda}-b^{2} x}\right) .
$$

Using the boundary conditions, we deduce that

$$
\begin{aligned}
& c_{1}=0, \quad \text { and } \\
& \lambda_{k}=\frac{1}{k^{2}+b^{2}}, \quad \text { for } k=0,1, \ldots, \infty
\end{aligned}
$$

Again, since $\lambda_{k}>0$, the singular values are $\sigma_{k}=\frac{1}{k^{2}+b^{2}}$. We omit finding the eigenfunctions since the decay rates of the singular values is the focus of this work.

The first 200 singular values for $A$ and $B$ are plotted in Figure 4.1 on a semi-log scale. There we see how the early values decay quadratically. This decay continues on for the infinitely many singular values approaching zero.

\subsection{Joint operator}

The joint operator $C$ will not be self adjoint since it does not have the same domain and codomain. i.e. $C: H \rightarrow H_{A} \oplus H_{B}$. Therefore we must compute the singular values, as opposed to the eigenvalues as done in the previous two examples. Namely, we solve 


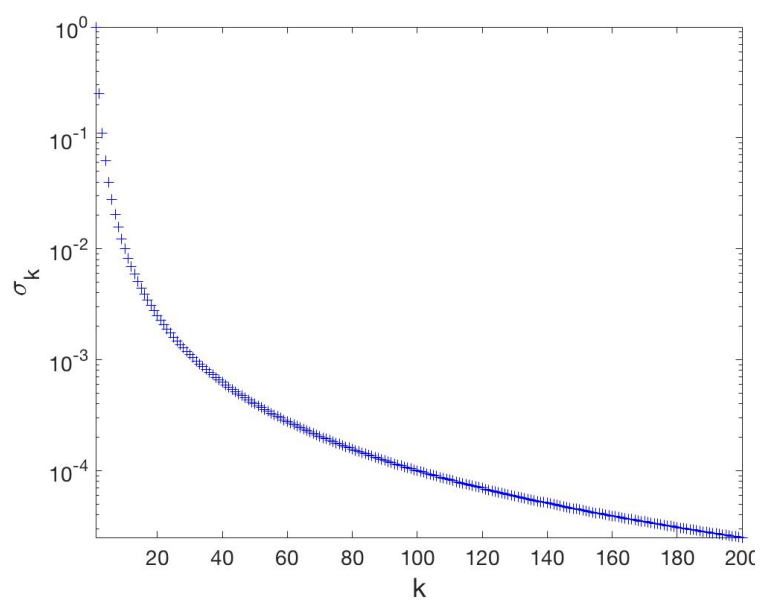

(a) $\sigma(A)$

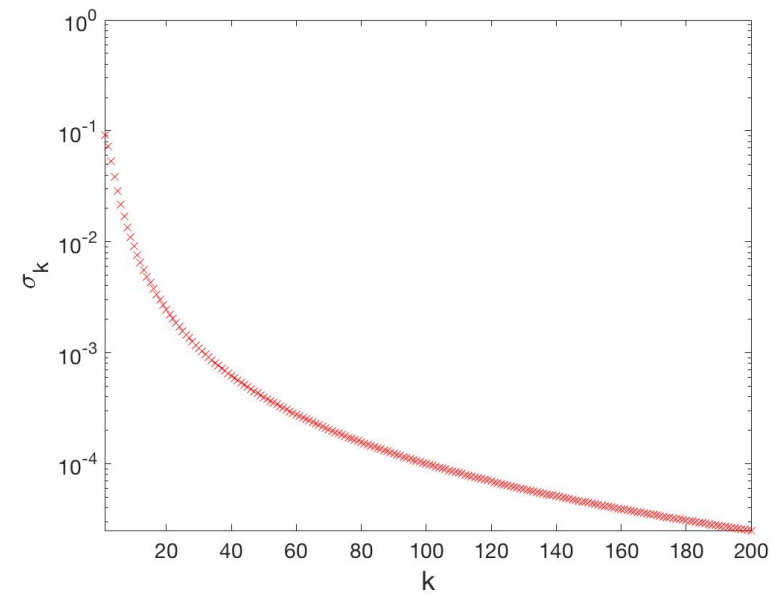

(b) $\sigma(B)$

Figure 4.1: Singular values of individual operators

$$
\begin{aligned}
\sigma^{2} \phi & =C^{*} C \phi \\
& =C^{*}(A \phi, B \phi) \\
& =A^{*} A \phi+B^{*} B \phi .
\end{aligned}
$$

Using the analytical approach to calculating the singular values in Section 3.2.2, we apply the differential operators and their adjoints to arrive at an ODE,

$$
\begin{aligned}
\sigma^{2}\left(L_{B} L_{B}^{*} L_{A} L_{A}^{*}\right) \phi & =\left(L_{B} L_{B}^{*}+L_{A} L_{A}^{*}\right) \phi \text { or }, \\
\sigma^{2}\left(\phi^{(8)}+2 b^{2} \phi^{(6)}+b^{4} \phi^{(4)}\right) & =\phi^{(4)}+2 b^{2} \phi^{(2)}+b^{4} \phi+\phi^{(4)} .
\end{aligned}
$$

As mentioned previously, some of our simplifications relied on the fact that $L_{A} L_{A}^{*}$ and $L_{B} L_{B}^{*}$ commute. We show that fact explicitly for this particular example.

Since the operators are self adjoint, $L_{A}^{*}$ is equivalent to $L_{A}$ and $L_{B}^{*}$ is equivalent to $L_{B}$. We will show that 


$$
L_{A} L_{A}^{*} L_{B} L_{B}^{*} h=L_{B} L_{B}^{*} L_{A}^{*} L_{A} h
$$

for all $h \in \mathcal{R}\left(A^{*} A\right)$. Let us first expand the left hand side of the equation.

$$
\begin{aligned}
L_{A} L_{A}^{*} L_{B} L_{B}^{*} h & =L_{A} L_{A}^{*} L_{B}\left(h^{(2)}+b^{2} h\right) \\
& =L_{A} L_{A}^{*}\left(h^{(4)}+2 b^{2} h^{(2)}+b^{4} h\right) \\
& =L_{A}\left(h^{(6)}+2 b^{2} h^{(4)}+b^{4} h^{(2)}\right) \\
& =h^{(8)}+2 b^{2} h^{(6)}+b^{4} h^{(4)} .
\end{aligned}
$$

Now, we expand the right hand side of the equation.

$$
\begin{aligned}
L_{B} L_{B}^{*} L_{A} L_{A}^{*} h & =L_{B} L_{B}^{*} L_{A}\left(h^{(2)}\right) \\
& =L_{B} L_{B}^{*}\left(h^{(4)}\right) \\
& =L_{B}\left(h^{(6)}+b^{2} h^{(4)}\right) \\
& =h^{(8)}+2 b^{2} h^{(6)}+b^{4} h^{(4)} .
\end{aligned}
$$

Therefore, we can see that

$$
L_{A} L_{A}^{*} L_{B} L_{B}^{*} h=L_{B} L_{B}^{*} L_{A}^{*} L_{A} h
$$

and so $L_{A} L_{A}^{*}$ and $L_{B} L_{B}^{*}$ commute.

Notice that the application of the differential operators at the previous steps imply the following boundary conditions: 


$$
\begin{gathered}
\phi(0)=\phi(\pi)=0, \\
\phi^{(2)}(0)=\phi^{(2)}(\pi)=0, \\
\phi^{(4)}(0)=\phi^{(4)}(\pi)=0, \\
\phi^{(6)}(0)=\phi^{(6)}(\pi)=0 .
\end{gathered}
$$

The singular function, singular value pairs $\left\{\left(\phi_{k}, \sigma_{k}\right)\right\}_{k=1}^{\infty}$ satisfy equation (4.1) and the corresponding boundary conditions. Even though this is a linear constant coefficient ODE, the eight roots of the characteristic polynomial make solving the boundary value problem analytically outside of the scope of this thesis. We therefore turn to the extension of the Galerkin method presented in section 3.2.3.

\subsection{Joint singular values}

The discretizations $A^{(n)}$ and $B^{(n)}$, described in Section 3.2.3, approximate the operators $A$ and $B$ with orthonormal bases. Following the approach in $[9,11]$, we use orthonormal box functions as our bases. The interval domains $\Omega_{s}=[0, \pi]$ and $\Omega_{t}=[0, \pi]$ are divided into $n$ subintervals $\left\{\Omega_{s}^{(i)}\right\}$ and $\left\{\Omega_{t}^{(i)}\right\}$ with equal lengths $h_{s}$ and $h_{t}$ respectively. The orthonormal box functions are then

$$
\begin{aligned}
& q_{i}(s)= \begin{cases}h_{s}^{-1 / 2}, & s \in \Omega_{s}^{(i)} \\
0, & \text { else }\end{cases} \\
& p_{j}(t)= \begin{cases}h_{t}^{-1 / 2}, & t \in \Omega_{t}^{(j)} \\
0, & \text { else }\end{cases}
\end{aligned}
$$

for $i, j=1, \ldots n$. Using these in equation (3.7) we have 


$$
a_{i j}^{(n)}=h_{s}^{-1 / 2} h_{t}^{-1 / 2} \int_{\Omega_{s}^{(i)}} \int_{\Omega_{t}^{(j)}} K_{A}(s, t) d t d s
$$

and similarly

$$
b_{i j}^{(n)}=h_{s}^{-1 / 2} h_{t}^{-1 / 2} \int_{\Omega_{s}^{(i)}} \int_{\Omega_{t}^{(j)}} K_{B}(s, t) d t d s .
$$

For $b=\pi$ the analytically derived singular values are plotted with the numerical approximations on a semi-log scale in Figure 4.2. It should be noted that the use of orthonormal box functions in this Galerkin process is most effective for smooth kernels, as sharp features cannot be fully resolved with finitely many boxes.

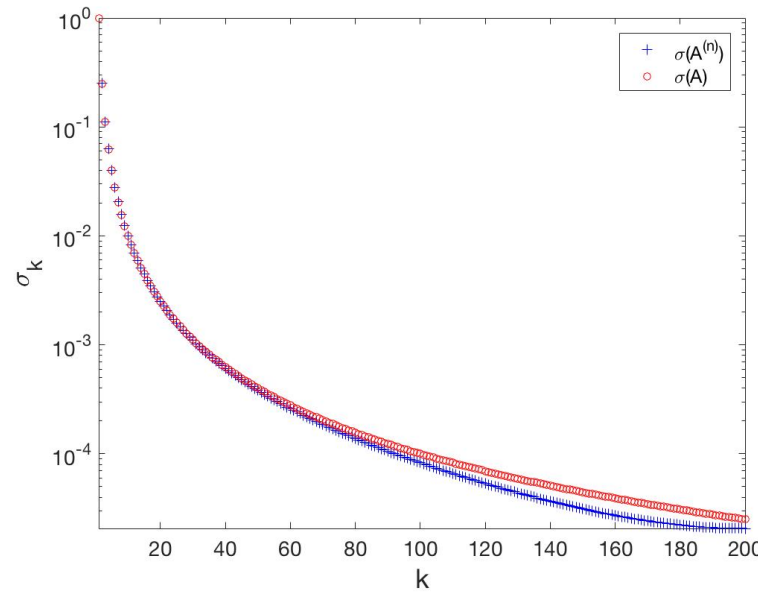

(a)

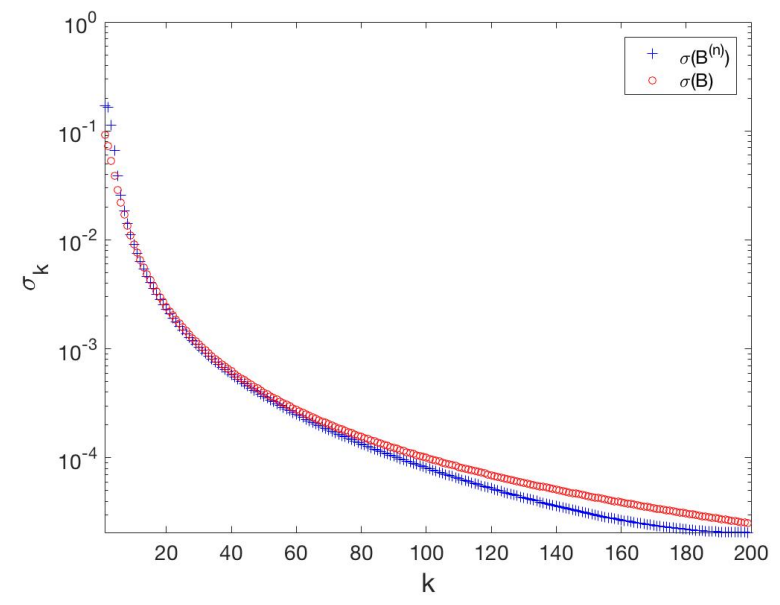

(b)

Figure 4.2: Analytical singular values and Galerkin method approximations using orthonormal box functions

The approximation $C^{(n)}$ to the joint operator $C=A \oplus B$ is formed by stacking the matrices $A^{(n)}$ and $B^{(n)}$. i.e.

$$
C^{(n)}=\left[\begin{array}{l}
A^{(n)} \\
B^{(n)}
\end{array}\right]
$$


We approximate the continuous singular values of $C$ with the discrete singular values of $C^{(n)}$. The values $\sigma\left(C^{(n)}\right)$ are shown in Figure 4.3 on both a semi-log and log-log scale. Note that the first several singular values have a decay rate that is less than quadratic. Eventually the later ones decay quadratically to zero, but if we wished to consider a truncated SVD solution, we could include more singular values than if we used either model $A$ or $B$ individually.

This modest improvement can clearly be seen in Figure 4.4, where we plot the singular values of the joint operator and singular values of the individual operators. We hypothesis that when we increase the complexity of the models under consideration there will be more substantial gains from joint inversion. We expect this to be especially true when considering models with complementary physics, such as ER and GPR.

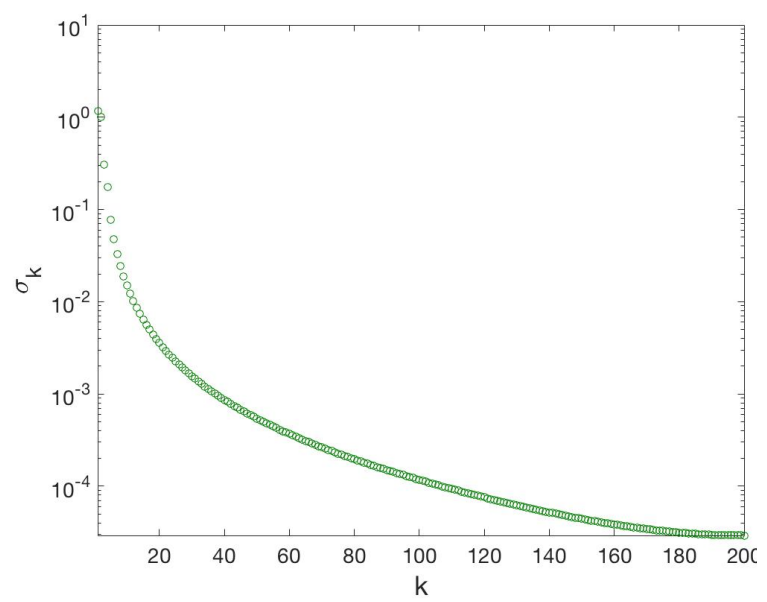

(a)

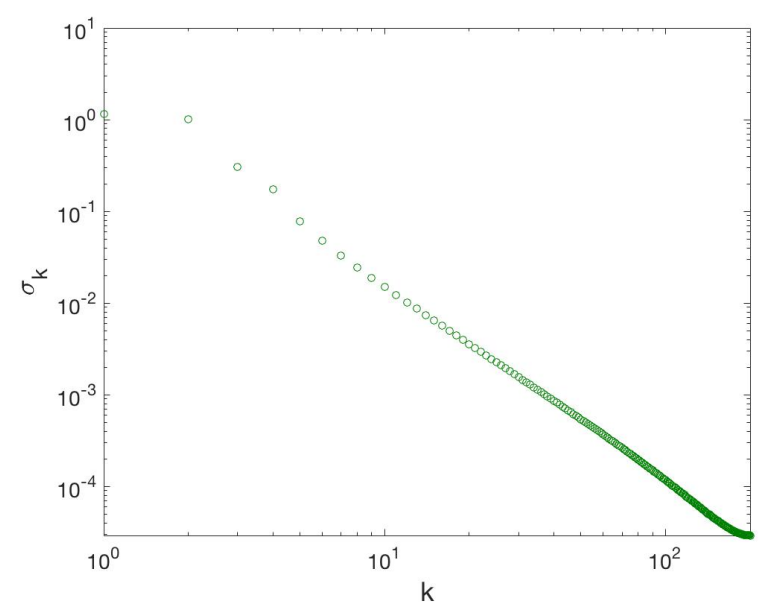

(b)

Figure 4.3: Approximate singular values of the joint operator $C$ on a semi-log scale (a), and a log-log scale (b)

Chapter 3 and 4 define and demonstrate the extension of singular value analysis of joint inversion problems to a continuous domain. This differs from the analysis 


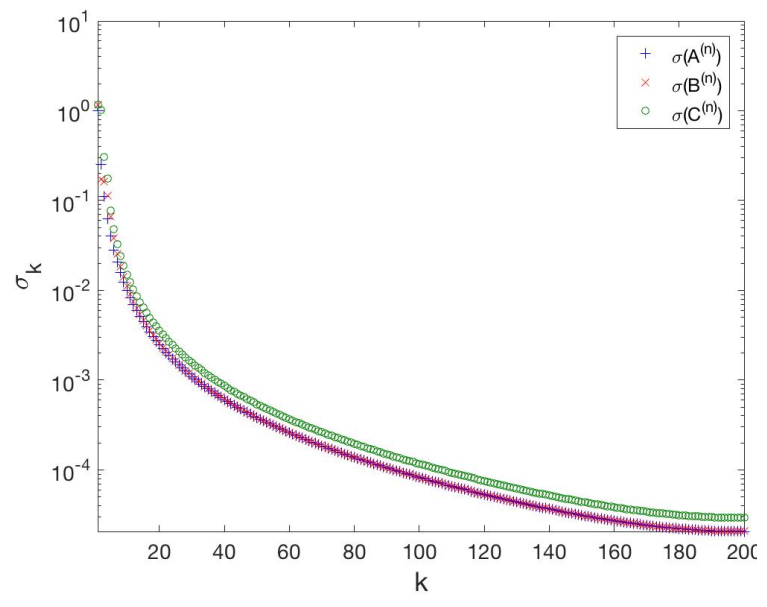

(a)

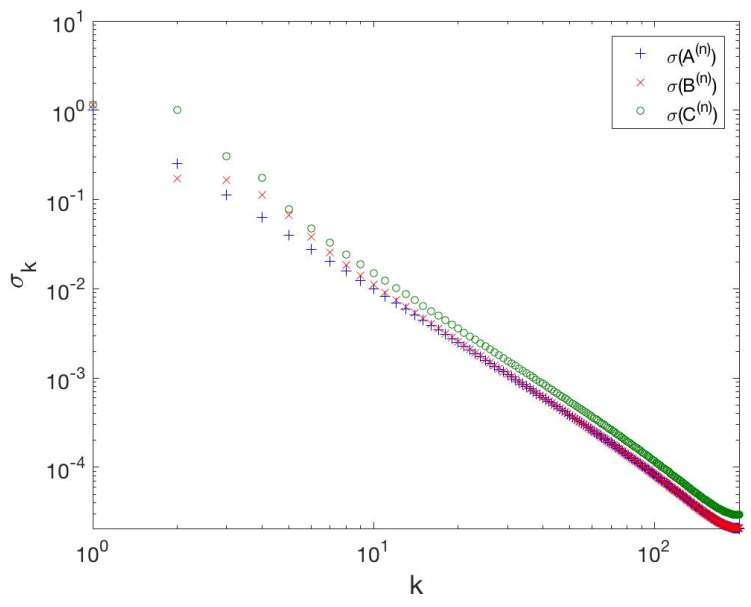

(b)

Figure 4.4: Approximate singular values of the individual operators $A$ and $B$, and the joint operator $C$ on a semi-log scale (a), and a log-log scale (b)

in [22], as there the authors began their study of ill-conditioning with the SVD of already discretized PDEs. The study of ill-posedness in this thesis depends instead on a discretization of the Green's functions which we have shown yields an approximation that converges to the SVE. To our knowledge, this has not been done previously for joint inversion. 


\section{CHAPTER 5}

\section{CONCLUSIONS AND FUTURE WORK}

In this thesis, we explored inverse problems in both discrete and continuous settings with a focus on ill-conditioning and ill-posedness, respectively. We saw that the primary tool for analysis of discrete linear problems, the singular value decomposition, had an extension to continuous problems with compact linear operators, the singular value expansion. Using the SVE we were able to quantify the ill-posedness of continuous inverse problems by the decay rate of the singular values.

We then considered joint inverse problems. The first mention of joint inversion came in [22], where the authors used the singular value decomposition to determine the degree of ill-conditioning in discrete inverse problems. The authors demonstrated in several examples that combining two models in a joint inversion, and effectively stacking discrete linear models, improved the conditioning of the problem.

We extended these notions to continuous inverse problems with compact linear operators. The analogous operation to stacking matrices is taking the direct sum of operators. We specifically considered the direct sum Green's function solutions to differential equations representing different types of data, and investigated the singular values of this joint operator. Analytical techniques for finding the singular values were presented. These could be useful for simple cases, however, numerical techniques will need to be used in general. To approximate the singular values of the 
joint operator, we extended the Galerkin method given in $[9,18]$. We the conditions necessary to capture the decay rate of the singular values by this approximation.

The results we have shown in this thesis are for simple cases. To increase the usefulness of the techniques presented here, we will need to construct more realistic and complicated models. The first step towards more realistic models will be the addition of another dimension. Green's function solutions for partial differential equations will naturally require at least two variables related to the dimension of the problem and two associated dummy variables. Therefore, we will need to further extend our numerical techniques.

As we are able to handle more complex models, we can work towards informing experimental design. Our intention is to mathematically test proposed joint inversion schemes based on Green's functions to see what will potentially be gained before discretizing the PDEs. It may not be the case that joint inversion will give substantial improvement over individual inversions. Therefore, these mathematical tests could help prevent unnecessary waste of both experimental and computational resources. 


\section{REFERENCES}

[1] Richard C Aster, Brian Borchers, and Clifford H Thurber. Parameter estimation and inverse problems. Elsevier Academic Press, Amsterdam, The Netherlands, 2005.

[2] J.B. Conway. A Course in Functional Analysis. Graduate Texts in Mathematics. Springer New York, 1994.

[3] Dean G Duffy. Green's Function with Applications. CRC Press, 2015.

[4] Andrew Geary. Setup of a dc resistivity survey, 2016.

[5] New Jersey Geological and Water Survey. Ground penetrating radar, 2005.

[6] Mark Gockenbach. Linear Inverse Problems and Tikhonov Regularization. The Mathematical Association of America, 2015.

[7] Mark S Gockenbach. Generalizing the gsvd. SIAM Journal on Numerical Analysis, 54(4):2517-2540, 2016.

[8] Paul Richard Halmos and Viakalathur Shankar Sunder. Bounded integral operators on L2 spaces. Ergebnisse Mathematik und GrenzGeb. Springer, Berlin, 1978.

[9] Per Christian Hansen. Computation of the singular value expansion. Computing, 40(3):185-199, 1988. 
[10] Per Christian Hansen. Rank-deficient and discrete ill-posed problems: numerical aspects of linear inversion, volume 4. Siam, 1998.

[11] Per Christian Hansen. Regularization tools version 4.0 for matlab 7.3. Numerical algorithms, 46(2):189-194, 2007.

[12] Per Christian Hansen. Discrete inverse problems: insight and algorithms. SIAM, 2010.

[13] Muneo Hori. Inverse analysis method using spectral decomposition of green's function. Geophysical Journal International, 147(1):77-87, 2001.

[14] John D Jackson. Classical electrodynamics. John Wiley \& Sons, Inc., New York, NY, 1999.

[15] Michel Kern. Numerical Methods for Inverse Problems. John Wiley \& Sons, 2016.

[16] Andreas Kirsch. An introduction to the mathematical theory of inverse problems, volume 120. Springer Science \& Business Media, 2011.

[17] Rainer Kress, V Maz'ya, and V Kozlov. Linear integral equations, volume 82. Springer, 1989.

[18] Rosemary A Renaut, Michael Horst, Yang Wang, Douglas Cochran, and Jakob Hansen. Efficient estimation of regularization parameters via downsampling and the singular value expansion. BIT Numerical Mathematics, pages 1-31, 2016.

[19] Bahaa Saleh. Introduction to subsurface imaging. Cambridge University Press, 2011. 
[20] Gilbert W Stewart. On the early history of the singular value decomposition. SIAM review, 35(4):551-566, 1993.

[21] Andre ̌ Nikolaevich Tikhonov, Vasiliı̆ IAkovlevich Arsenin, and Fritz John. Solutions of ill-posed problems, volume 14. Winston Washington, DC, 1977.

[22] K Vozoff and DLB Jupp. Joint inversion of geophysical data. Geophysical Journal International, 42(3):977-991, 1975.

[23] Michael S Zhdanov. Inverse theory and applications in geophysics, volume 36. Elsevier, 2015. 